\title{
Metal-rich absorbers at high redshifts: abundance patterns ${ }^{\star}$
}

\author{
S. A. Levshakov ${ }^{1,2}$, I. I. Agafonova ${ }^{2}$, P. Molaro ${ }^{1}$, D. Reimers ${ }^{3}$, and J. L. Hou ${ }^{4}$ \\ 1 INAF - Osservatorio Astronomico di Trieste, via G. B. Tiepolo 11, 34131 Trieste, Italy \\ 2 Ioffe Physical-Technical Institute, Polytekhnicheskaya Str. 26, 194021 St. Petersburg, Russia \\ e-mail: lev@astro.ioffe.rssi.ru \\ 3 Hamburger Sternwarte, Universität Hamburg, Gojenbergsweg 112, 21029 Hamburg, Germany \\ 4 Key Lab. for Research in Galaxies and Cosmology, Shanghai Astronomical Observatory, CAS, 80 Nandan Road, Shanghai 200030, \\ PR China
}

Received 29 March 2009 / Accepted 27 August 2009

\section{ABSTRACT}

\begin{abstract}
Aims. To study chemical composition of metal-rich absorbers at high redshifts in order to understand their nature and to determine sources of their metal enrichment.

Methods. From six spectra of high- $z$ QSOs, we select eleven metal-rich, $Z \gtrsim Z_{\odot}$, and optically-thin to the ionizing radiation, $N(\mathrm{HI})$ $<10^{17} \mathrm{~cm}^{-2}$, absorption systems ranging between $z=1.5$ and $z=2.9$ and revealing lines of different ions in subsequent ionization stages. Computations are performed using the Monte Carlo inversion (MCI) procedure complemented with the adjustment of the spectral shape of the ionizing radiation. This procedure along with selection criteria for the absorption systems guarantee the accuracy of the ionization corrections and of the derived element abundances $(\mathrm{C}, \mathrm{N}, \mathrm{O}, \mathrm{Mg}, \mathrm{Al}, \mathrm{Si}, \mathrm{Fe})$.

Results. The majority of the systems (10 from 11) show abundance patterns which relate them to outflows from low and intermediate mass stars. One absorber is enriched prevalently by SNe II, however, a low percentage of such systems in our sample is conditioned by the selection criteria. All systems have sub-kpc linear sizes along the line-of-sight with many less than $\sim 20 \mathrm{pc}$. In several systems, silicon is deficient, presumably due to the depletion onto dust grains in the envelopes of dust-forming stars and the subsequent gasdust separation. At any value of $[\mathrm{C} / \mathrm{H}]$, nitrogen can be either deficient, $[\mathrm{N} / \mathrm{C}]<0$, or enhanced, $[\mathrm{N} / \mathrm{C}]>0$, which supposes that the nitrogen enrichment occurs irregularly. In some cases, the lines of $\mathrm{Mg}$ II $\lambda \lambda 2796,2803$ appear to be shifted, probably as a result of an enhanced content of heavy isotopes ${ }^{25} \mathrm{Mg}$ and ${ }^{26} \mathrm{Mg}$ in the absorbing gas relative to the solar isotopic composition. Seven absorbers are characterized by low mean ionization parameter $U, \log U<-2.3$, among them only one system has a redshift $z>2\left(z_{\text {abs }}=2.5745\right)$ whereas all others are found at $z \sim 1.8$. This statistics is not affected by any selection criteria and reflects the real rise in number of such systems at $z<2.0$. Comparing the space number density of metal-rich absorbers with the comoving density of star-forming galaxies at $z \sim 2$, we estimate that the circumgalactic volume of each galaxy is populated by $\sim 10^{7}-10^{8}$ such absorbers with total mass $\lesssim 1 / 100$ th of the stellar galactic mass. Possible effects of high metal content on the peak values of star-forming and AGN activities at $\tilde{z} \sim 2$ are discussed.
\end{abstract}

Key words. line: formation - line: profiles - galaxies: abundances - intergalactic medium - quasars: absorption lines cosmology: observations

\section{Introduction}

The majority of metal-bearing absorbers detected in quasar spectra demonstrate very low metallicity of about $1 / 30$ of the solar value, $Z_{\odot}$, and below (Songaila \& Cowie 1996; Simcoe et al. 2004). Compared to the metal-poor absorbers, the systems with high metallicity - from solar to oversolar - occur in quasar spectra quite seldom. A particular interest to the metal-rich absorbers stems from the fact that high metallicities are usually measured in the central parts of galaxies (Rich et al. 2007; Cohen et al. 2008; Zoccali et al. 2008; Davies et al. 2009) and are closely related to the starburst activity and chemical evolution of AGNs/QSOs (Hamann \& Ferland 1999; D’Odorico et al. 2004; Polletta et al. 2008; Silverman et al. 2009). At high redshifts, the only way to gain an insight into the chemical composition of gas distributed over large cosmological distances is through the analysis of absorption systems in quasar spectra. Individual element abundances, or abundance patterns, derived from the metal-rich systems can be used to reconstruct their chemical history and to estimate relative contributions from $\mathrm{SNe}$ II, SNe Ia, and AGB-stars.

\footnotetext{
* Based on observations obtained with the UVES at the VLT Kueyen telescope (ESO, Paranal, Chile).
}

The luminous quasar space density distribution (Fan et al. 2001) and observations of high-redshift galaxies (Dickinson et al. 2003) show that the redshift $z \sim 2$ represents a turning point in the global cosmic evolution. The study of the chemical enrichment at different $z$ can lead to important clues concerning the factors which cause the peak values of AGN and star-forming activities at $z \sim 2$.

To obtain chemical enrichment patterns of the absorbing gas, accurate abundances of different elements are required. Abundance measurements in high-metallicity damped Ly- $\alpha$ absorbers (DLAs) and sub-DLAs where ionization corrections are supposed to be small are limited either to low abundant species like $\mathrm{Zn}, \mathrm{Mn}$, and $\mathrm{Cr}$, or to those which have transitions with low oscillator strengths like Fe and Si since heavily saturated lines of other elements prevent their accurate column density determination (Prochaska et al. 2006; Péroux et al. 2006; Meiring et al. 2007; Meiring et al. 2008; Péroux et al. 2008). However, just $\mathrm{Fe}$ and $\mathrm{Si}$ (as a proxy for $\alpha$-elements) which belong to the key species in the abundance pattern, can be severely depleted (Hou et al. 2001; Vladilo 2002; Vladilo \& Péroux 2005; Quast et al. 2008), thus hampering significantly the identification of the enrichment agents. Note that metal-rich DLAs are very rare - up to now, only about a dozen is detected. 
On the other hand, in absorbers which are optically-thin to the ionizing radiation ${ }^{1}$ the ionization correction factors are large and, consequently, the resulting element abundances are very sensitive to their values. In general, ionization corrections are determined by the ionization parameter $U$ and by the spectral shape of the ionizing radiation. To increase the reliability of the measured abundances both a special selection of absorption systems and an adequate mathematical treatment of the inverse spectroscopy problem are required.

An approach employed in the present paper is based on the physical model of absorbing gas which assumes fluctuating gas density and velocity fields inside the absorber. This model is a generalization of a commonly used approximation of a planeparallel gas slab of uniform density and a microturbulent treatment of the velocity field. The corresponding computational procedure used to calculate the metal abundances includes also the adjustment of the spectral shape of the ionizing radiation (see Sect. 2).

Since relative element ratios can differ from the solar pattern, it should be clarified on base of which species we conclude whether the gas is metal-rich or not. Metallicity in stars is usually characterized by the iron content, that of strong absorption systems (LLSs and DLAs) - by the content of zinc or sulfur which are not condensed significantly into dust. In optically-thin absorption systems, metallicity is conventionally estimated on base of carbon. Thus, in the present metal-rich sample we included absorbers with carbon abundances near and above the solar value, $(\mathrm{C} / \mathrm{H})_{\odot}=2.45 \times 10^{-4}$ (Asplund et al. 2004).

The selection of systems occurred in the following way. In the framework of our project to study the spectral energy distribution (SED) of the ionizing radiation the quasar spectra were searched for the optically-thin absorption systems revealing lines of different ions (Agafonova et al. 2005). The presence of at least one pair of ions of the same element (e.g. C II, C IV) was essential for the system to be included in the sample. These subsequent ions ensure that the mean ionization parameter can be estimated without any additional assumptions. In total, about 50 absorbers identified in high-resolution spectra of $\sim 20$ QSOs with emission redshifts $1.7<z<4.5$ were selected and analyzed. The derived metallicities range from $1 / 1000$ to several times solar values. A part of results was described in our publications dealing with the evolution of the background SED with redshift (Reimers et al. 2006; Agafonova et al. 2007) and the restoration of the SED of the outcoming quasar radiation on base of associated systems (Reimers et al. 2005; Levshakov et al. 2008, hereafter Paper I). These publications included already several systems with high metal contents, however, enrichment scenarios were not elaborated. This is just what the present paper is focused on where we consider additional 7 metal-rich absorbers. With the previously described systems, the sample of metal-rich absorbers consists now of 11 systems with redshifts ranging from $z=1.5$ to $z=2.9$.

\section{Computational method}

Absorption systems are analyzed by means of the Monte Carlo inversion (MCI) procedure described in Levshakov et al. (2000, hereafter LAK), with further details in Levshakov et al. (2003). This procedure supposes that all lines observed in a metal system are formed in the gas slab with fluctuating density $n_{\mathrm{H}}(x)$ and velocity $v(x)$ fields (here $x$ is the space coordinate along the line-ofsight within the absorber). Further assumptions are that within

${ }^{1}$ Column density of neutral hydrogen $N(\mathrm{H} \mathrm{I})<3 \times 10^{17} \mathrm{~cm}^{-2}$. the absorber the metal abundances are constant and the gas is in thermal and ionization equilibrium. The procedure is applicable for optically-thin systems. The inputs are the observed line profiles and the ionization curves for each ion (hydrogen + metals) included in the analysis. The ionization curves (the dependence of the ion fraction on the ionization parameter $U$ ) are computed with the photoionization code CLOUDY version 07.02.01 (last described by Ferland et al. 1998) which in turn uses as the inputs an ionizing continuum and a set of element abundances. The fitting parameters (outputs) of the MCI procedure are: the mean ionization parameter $U_{0}$, the total hydrogen column density $N_{\mathrm{H}}$, the line-of-sight velocity dispersion, $\sigma_{v}$, and the density dispersion, $\sigma_{y}$, of the bulk material $\left[y \equiv n_{\mathrm{H}}(x) / n_{0}\right]$, and the contents $Z_{a}$ of metals included in the objective function [Eqs. (29), (30) in LAK]. The fitting parameters are estimated by minimization of residuals between synthetic and observed line profiles. As in all Monte Carlo methods, integrals [here the intensity at a point within the line profile, see Eq. (12) in LAK] are calculated by modeling the distribution of the integrated function. This function is a convolution of the gas density and the local absorption coefficient (which depends on the velocity through the irregular Doppler shifts) and, hence, the integrated function is realized through the density and velocity distributions over the integration path. Both the distributions of $n_{\mathrm{H}}(x)$ and $v(x)$ are represented by their sampled values $\left\{n_{i}\right\}$ and $\left\{v_{i}\right\}$ at equally spaced points $x_{i}$ along the line-of-sight, and optimal configurations of $\left\{n_{i}\right\}$ and $\left\{v_{i}\right\}$ are estimated by the simulated annealing algorithm. With the estimated values of the fitting parameters and the distributions of $n_{\mathrm{H}}(x)$ and $v(x)$, we can calculate the column densities of all ions (Eqs. (24), (25) in LAK) and the mean values for the kinetic temperature, $T_{\text {kin }}$, the gas density, $n_{0}$, and the linear size, $L$, of the absorber along the line-of-sight (see below).

If the metal abundances $Z_{a}$ obtained from the MCI procedure differ from those used in CLOUDY to calculate the ionization curves, then these curves are re-calculated with the updated abundances and the MCI procedure is run again. The iterations stop after the concordance between the abundances used in CLOUDY and derived from MCI is reached.

At this stage of calculations, the shape (SED) of the ionizing radiation (which comes into the MCI procedure through the ionization curves) is treated as an external parameter, i.e., it is keeping constant. In case when a first guessed SED cannot provide an acceptable solution, a complemented computational routine aimed at adjusting the spectral shape is applied. The corresponding algorithm based on the response surface methodology from the theory of experimental design is described in detail in Agafonova et al. (2005, 2007). It includes a parameterization of the SED by means of a set of factors and the estimation of their values in a way to ensure that all obtained physical parameters are self-consistent and all observed profiles are described with a sufficient accuracy (i.e., $\chi^{2} \sim 1$ ).

It is known from both model calculations (Haardt \& Madau 1996; Fardal et al. 1998; Madau \& Haardt 2009) and from SEDs restored from observational data (Agafonova et al. 2005, 2007) that spectral shapes of the intergalactic ionizing background in the energy range $E>1$ Ryd have complex forms with an emission bump at $E \lesssim 3$ Ryd and a stepwise break at $E>3$ Ryd. However, as noted in Agafonova et al. (2007; Sect. 3.1.2), at high metallicities the dependence of the ion fractions on the absolute abundances (especially on the contents of the most abundant elements such as carbon and oxygen) is comparable or even overrides the dependence on the spectral shape. For the systems considered in the present study this means that the inherent uncertainty in the values of the absolute element abundances 
makes it impossible to estimate the SED of the underlying ionizing continuum in full detail. That is why the spectral shape is represented and estimated in a simplified form including only 2 factors: a power law index $\alpha\left(v^{-\alpha}\right)$ in the range $1<E<4$ Ryd, and a depth of a break at 4 Ryd. The power law index for the energy range beyond this break ( $E>4$ Ryd) was fixed as $1-0.8 \alpha$.

The mean linear size $L$ of an absorbing cloud along the line of sight can be estimated as $L=N_{\mathrm{H}} / n_{0}$, where $N_{\mathrm{H}}$ is the total hydrogen column density, and $n_{0}$ is the mean gas density of the absorber. The mean gas density is calculated from the mean ionization parameter $U_{0}$ and the density dispersion $\sigma_{y}(\mathrm{LAK})$ :

$n_{0}=\frac{n_{\mathrm{ph}}}{U_{0}} \mathrm{e}^{\sigma_{y}^{2}}$

Here $n_{\mathrm{ph}}$ is the number density of photons (in units of $\mathrm{cm}^{-3}$ ) with energies above 1 Ryd

$n_{\mathrm{ph}}=\frac{4 \pi}{c h} J_{912} \int_{v_{c}}^{\infty}\left(\frac{J_{v}}{J_{912}}\right) \frac{\mathrm{d} v}{v}$,

where $c, h, v_{c}$, and $J_{v}$ are the speed of light, the Planck constant, the frequency of the hydrogen Lyman edge, and the specific intensity (in ergs $\mathrm{cm}^{-2} \mathrm{~s}^{-1} \mathrm{sr}^{-1} \mathrm{~Hz}^{-1}$ ). $J_{912}$ is the normalizing value and represents the intensity of the ionizing radiation at $912 \AA$ ( 1 Ryd). As a reference, the value of $J_{912}$ for the intergalactic background at a corresponding redshift is taken from Haardt \& Madau (1996).

If a strong radiation source such as a quasar is located close (up to $\sim 2 \mathrm{Mpc}$ away) to the absorber, then the intensity $J_{912}$ of the incident ionizing radiation exceeds significantly the mean intergalactic level (see, e.g., examples in Paper I). Thus, the gas density/linear size calculated on base of the intergalactic $J_{912}$ represents in fact only a lower/upper limit, respectively.

A few systems from the present study show signs of the incomplete coverage of the background light source. Computational treatment of such systems occurred as described in Sect. 2 of Paper I. The covering factor is assumed to be the same for the whole cloud, i.e. it does not depend on $\lambda$ (or, equivalently, on the radial velocity $v$ ). However, different covering factors are allowed for different ions. This follows from a model of the absorption arising in a gas cloud with fluctuating density. Namely, ions of higher ionization stages trace rarefied gas which can be quite extended (i.e., covers a greater part of the background source) whereas low ionization ions originate in more dense and, hence, compact volumes (i.e., the covering factor is smaller).

The laboratory wavelengths and oscillator strengths for all lines except Si III were taken from Morton (2003). For Si III Morton gives 1206.500 ̊ (see comments on page 190 in Morton 1991), however with this wavelength the Si III line comes out shifted by $\sim 2.5 \mathrm{~km} \mathrm{~s}^{-1}$ relative to both $\mathrm{Si}$ II and Si IV lines. Since metal lines in absorption systems considered in the present paper are very narrow $\left(F W H M \lesssim 15 \mathrm{~km} \mathrm{~s}^{-1}\right)$ this shift becomes noticeable. On the other hand, other catalogs of atomic and ionic spectral lines (e.g., Bashkin \& Stoner 1975; Kelly 1987) and catalogs of solar lines (e.g., Sandlin et al. 1986; Curdt et al. 2001) give for Si III the wavelength $1200.51 \AA$. This value fits much better to what is observed in the absorption systems and it was therefore adopted for the present work.

Solar abundances were taken from Asplund et al. (2004). Note that their solar abundance of nitrogen, $(\mathrm{N} / \mathrm{H})_{\odot}=6 \times 10^{-5}$, is $\sim 1.4$ times (0.15 dex) lower than that from Holweger (2001).

Quasar spectra were obtained with the UVES/VLT in the framework of the ESO Large Program "QSO Absorption Line
Systems" (ID No. 166.A-0106). Data reduction was performed by B. Aracil (Aracil et al. 2004). Except Q0329-385, all QSOs used in the present paper have been discovered in course of the Hamburg/ESO survey (Wisotzki et al. 1996; Reimers et al. 1996; Wisotzki et al. 2000).

\section{Analysis of individual systems}

\subsection{Quasar HE1347-2457}

\subsubsection{System at $\mathrm{z}_{\mathrm{abs}}=2.5745$}

The system at $z_{\mathrm{abs}}=2.5745$ consists of the neutral hydrogen Lyman series lines $\left(\mathrm{L}_{1}-\mathrm{L}_{10}\right)$ and many lines of different metal ions (Fig. 1). Expected positions of the Fe II $\lambda \lambda 2382.76,2600.17$ lines coincide with strong telluric absorptions, Fe III $\lambda 1122.52$ falls in a noisy part of the spectrum and cannot be extracted from the noise. The emission redshift of the quasar HE13472457 is $z_{\mathrm{em}}=2.578$ (based on C II emission), so that the absorber is detached by only $300 \mathrm{~km} \mathrm{~s}^{-1}$ from the quasar. The profiles of the OVI $\lambda \lambda 1031,1037$ absorption lines differ from the profiles of other ionic transitions all of which exhibit similar line shapes. The observed intensities of low ionization (C II, Si II) and high ionization (C IV, Si IV) lines do not differ from each other significantly implying a rather low ionization parameter $U_{0}, \log \left(U_{0}\right)<-1.7$, for all types of the incident ionizing spectra. At such $U_{0}$, a considerable amount of O VI can hardly be produced. Thus, this absorption system originates probably in a dense and relatively cold gas (seen in lines of C II-C IV, Si II-Si IV, N III, Mg II, and Al III) embedded in a hot and highly ionized medium seen in O VI and H I $\lambda \lambda 1215,1025$ lines.

The absorption profiles of the C III $\lambda 977$ and HI $\lambda 1025$ lines $^{2}$ have flat bottoms and reveal non-zero residual intensities at the line centers ( 0.02 and 0.01 , respectively) pointing to the incomplete coverage of the background light source, i.e. the covering factor for $\mathrm{C}$ III is $C(\mathrm{C} \mathrm{III})=0.98$ and for hydrogen $C(\mathrm{HI})=0.99$. Due to the detection of many lines of neutral hydrogen its column density can be estimated quite accurately: $N(\mathrm{H} \mathrm{I})=(9.5 \pm 0.3) \times 10^{14} \mathrm{~cm}^{-2}$. A clear (unblended) doublet of C IV $\lambda \lambda 1548,1550$ allows us to estimate $C(\mathrm{C}$ IV $)=0.99$ and $N(\mathrm{C}$ IV $)=(3.2 \pm 0.1) \times 10^{13} \mathrm{~cm}^{-2}$. Because of the line blending, low $\mathrm{S} / \mathrm{N}$ and/or the presence of a single unsaturated line only (like Si III $\lambda 1206$ and N III 2989 ) accurate covering factors for other ions cannot be determined. Their limiting values are as follows: $0.98>C\left(\mathrm{C}_{\text {II }} / \mathrm{Si}\right.$ II $/ \mathrm{Mg}$ II $)>0.6,0.98>$ $C(\mathrm{Si}$ III/N III/Al III $)>0.7$, and $0.98>C(\mathrm{Si} \mathrm{IV})>0.8$. We note that covering factors may be slightly different for each of the two components comprising the absorption system under consideration.

With such uncertainties in covering factors the shape of the ionizing spectrum cannot be restored uniquely. Nevertheless, some conclusions about the SED can be obtained. The incomplete covering of the background light source indicates that the system is located close to the quasar. Common first guess for the SED of the outcoming quasar radiation is a power law, $v^{-\alpha}$. However, pure power law spectra are not consistent with the observed line intensities. Namely, for all tried spectral indices $\alpha$, $-2.0<\alpha<-0.5$, the mean ionization parameter $U_{0}$ is low and

\footnotetext{
2 The H I $\lambda 1215$ line is black because of blending with Ly- $\alpha$ from the highly ionized system. An appropriate $N(\mathrm{HI})$ for this system is $\sim 10^{14} \mathrm{~cm}^{-2}$, i.e. its Ly- $\alpha$ is saturated but Ly- $\beta$ is not.
} 


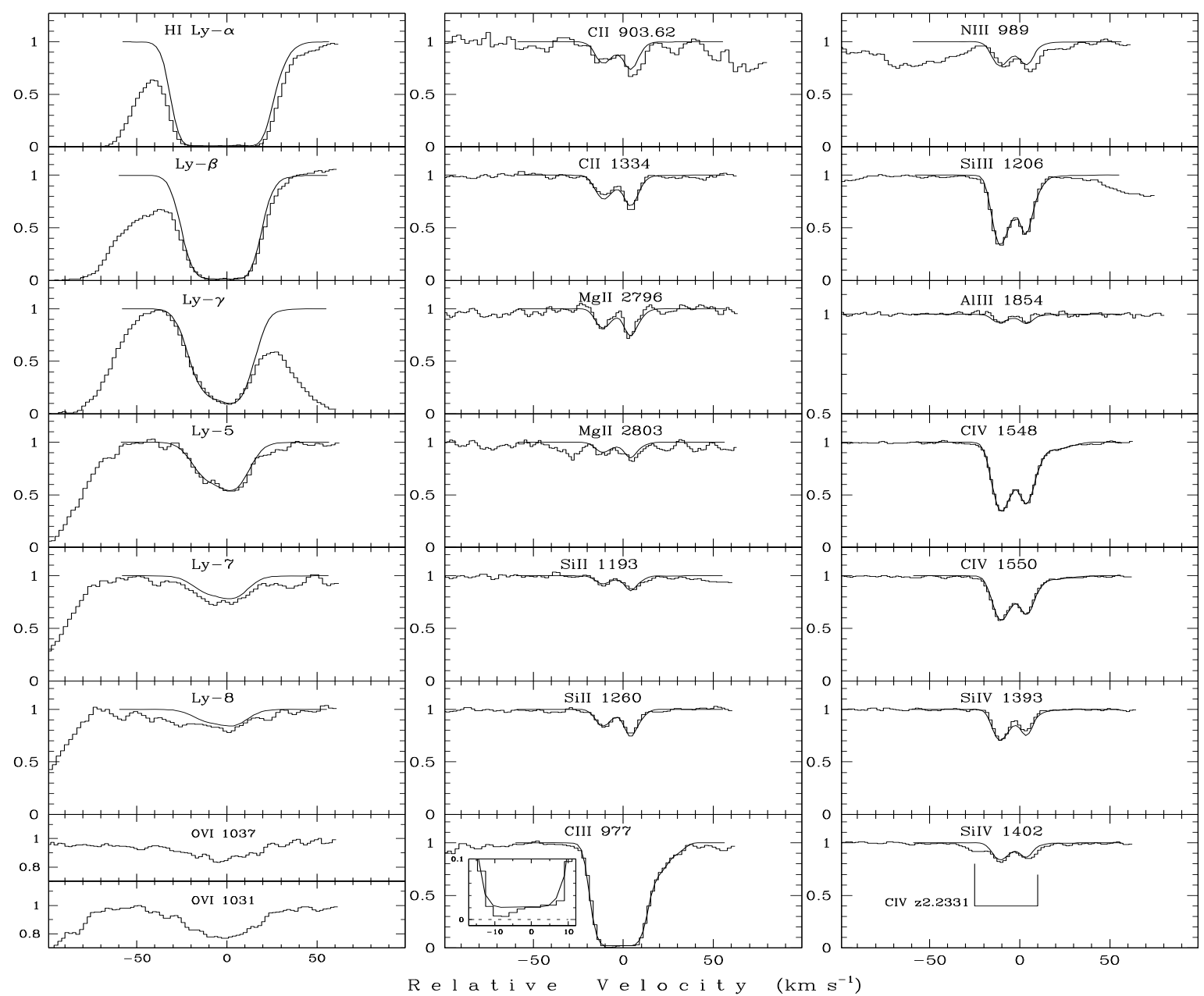

Fig. 1. Hydrogen and metal absorption lines from the $z_{\text {abs }}=2.5745$ system towards HE1347-2457 (solid-line histograms). The vertical axis is normalized intensities. The zero radial velocity is fixed at $z=2.5745$. Synthetic profiles are plotted by the smooth curves. The wide profiles of the O VI $\lambda \lambda 1031,1037$ lines show that the narrower line metal-rich system is embedded in a hot gas (seen also in H I Ly- $\alpha$ and Ly- $\beta$ lines as a difference between the observed and synthetic profiles). The insert in panel C III $\lambda 977$ demonstrates a non-zero residual intensity at the line center. The identified blend is marked in panel Si IV $\lambda 1402$.

the corresponding fraction of C IV is low as well, which makes the abundance of carbon,

$\mathrm{C} \sim \frac{\mathrm{C}_{\mathrm{IV}}}{\mathrm{H}_{\mathrm{I}}} \frac{\Upsilon_{\mathrm{HI}}}{\Upsilon_{\mathrm{CIV}}}$,

to be very high, 4-5 times solar value (and the abundance of magnesium even higher). With such a high metal content it is impossible to describe the observed profiles of hydrogen lines: in particular the central parts of the synthetic profiles of the $\lambda 972.53$ and $\lambda 937.80$ lines look too wavy following the pattern exhibited by metal ions. In order to decrease the metallicity, the ratio $\Upsilon_{\mathrm{H}_{\mathrm{I}}} / \Upsilon_{\mathrm{C}_{\text {IV }}}$ should be lowered, and this occurs when the observed profiles of $\mathrm{C} \mathrm{III/C} \mathrm{IV} \mathrm{and} \mathrm{Si}$ II/Si III/Si IV lines are described with higher values of $U_{0}$. As it was the case with the associated systems from Paper I, to fit to these conditions the ionizing spectra should have a break at 4 Ryd. The depth of the break depends on the adopted covering factors, but the intensity decrease of at least one order of magnitude at $E>4 \mathrm{Ryd}$ is required. On the other hand, a too deep drop of the intensity (the depth of the break $>1.3$ dex) leads to the overproduction of Si III compared to the observed profile even at the lower limit of $C$ (Si III). A small difference between the C IV/C II and Si IV/Si II ratios makes preferable the power indices between 1 and 4 Ryd of $\alpha \sim 1.2-1.6$.

As already noted, at high metallicities (above solar) the relative element contents, especially those of the most abundant elements carbon and oxygen, affect quite strongly the calculated ion fractions (upon which the procedure of spectral shape estimation is based). Lines of different oxygen ions are not available in the $z_{\text {abs }}=2.5745$ system, and the unknown oxygen content gives an additional degree of freedom in the procedure of the spectral shape estimation. These considerations restrict the acceptable spectral shapes as shown by the shaded area in Fig. 2. The values of the physical parameters obtained with the MCI routine are given in Tables 1-3. The column densities in Table 2 are calculated with the upper limits of the covering factors, i.e. real column densities may be up to $50 \%$ higher if, for instance, $C \simeq 0.6$. The gas density of $5 \times 10^{-3} \mathrm{~cm}^{-3}$, and the linear size of $25 \mathrm{pc}$ given in Table 1 are estimated with the mean intergalactic value of $J_{912}$. However, for an absorber located close to the quasar the intensity of the ionizing continuum $J_{912}$ will be enhanced making the corresponding gas density higher and the linear size smaller. For example, $J_{912}$ in the vicinity of the associated system at $z_{\mathrm{abs}}=2.1470$ towards HE0141-3932 (Sect. 2.2.1 


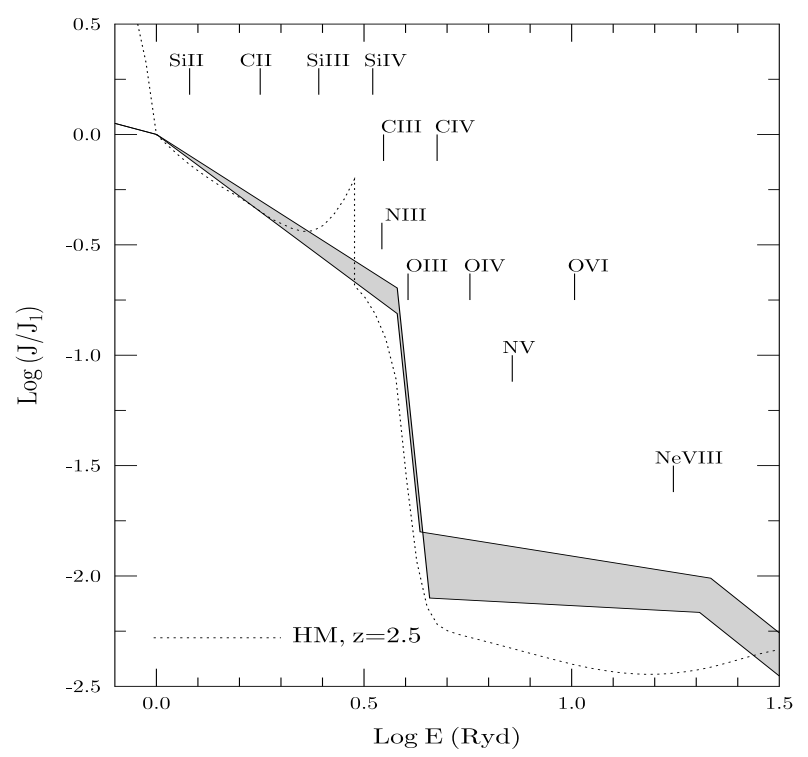

Fig. 2. The ionizing spectrum corresponding to the ionization state observed in the $z_{\text {abs }}=2.5745$ system (HE1347-2457) is one from the shaded area. For comparison the intergalactic ionizing spectrum at $z=2.5$ modeled by Haardt \& Madau (1996) is shown by the dotted line. The spectra are normalized so that $J_{v}(h v=1 \mathrm{Ryd})=1$. The positions of ionization thresholds of different ions are indicated by tick marks.

in Paper I) was enhanced by two orders of magnitude as compared to the mean intergalactic level. With such an intensity the linear size of the present absorber would be of a sub-parsec scale.

In spite of all uncertainties inherent to the present system, the relative abundance ratios retain a quite stable pattern (Table 3 ). Namely, the abundances of carbon, magnesium and aluminium are strongly oversolar reaching $2.5-3$ solar values whereas silicon and nitrogen are considerably underabundant compared to carbon (by $0.2-0.4$ dex depending on the shape of the ionizing spectrum and the adopted covering factors for silicon and nitrogen ions).

The derived underabundance of nitrogen to carbon, [N/C] $<-0.2$, at highly oversolar metallicity does not comply with models of chemical evolution of gas in the central parts of QSOhosting galaxies (Hamann \& Ferland 1999) and models of galactic chemical enrichment (Calura \& Matteucci 2004) all of which predict significant overabundance of nitrogen relative to other elements at metallicities above solar. However, nitrogen measurements in planetary nebulae show that even at high metallicities nitrogen can be both under- and overabundant (Aller \& Czyzak 1983; Perinotto 1991; Richer \& McCall 2008). Low ratios [N/C] $<0$ at near-solar metallicities were reported also for intergalactic absorbers by Reimers et al. (2005) and Jenkins et al. (2005).

Quite peculiar in the abundance pattern of the system under study is its low content of silicon. In optically-thin quasar absorbers the standard finding is $[\mathrm{Si} / \mathrm{C}]>0$ which is attributed to the SNe Type II stars as the main source of silicon enrichment (Songaila \& Cowie 1996; Simcoe et al. 2006). In the $z_{\mathrm{abs}}=$ 2.5745 system the contents of $\mathrm{C}, \mathrm{Mg}$ and $\mathrm{Al}$ follow the solar pattern, thus we can conclude that it is not carbon which is enhanced but silicon which is in fact deficient. Taking into account high metallicity of the absorbing gas, it is conceivable to assume that this deficit is caused by depletion of silicon into dust. It is well known that convective envelopes of post-main sequence stars (Red Giants, Red Supergiants and Asymptotic
Giant Branch stars) as well as planetary nebulae forming around these stars are places of the effective dust condensation. In the oxygen-rich environment, $\mathrm{C} / \mathrm{O}<1$, the main dust species are iron and silicates consisting of olivine $\mathrm{Mg}_{2 x} \mathrm{Fe}_{2(1-x)} \mathrm{SiO}_{4}$, pyroxen $\mathrm{Mg}_{x} \mathrm{Fe}_{1-x} \mathrm{SiO}_{3}$ and quartz $\mathrm{SiO}_{2}$ (Gail \& Sedlmayr 1999; Jeong et al. 2003; Ferrarotti \& Gail 2006). Proportions of olivine, pyroxen and quartz among the silicate dust grains are unknown, thus magnesium could also be depleted along with silicon and oxygen. However, there are reasons to suppose that depletion of magnesium - if any - is in fact much less pronounced than that of silicon (see Sect. 4.2).

Taking into account high metallicity, relative element ratios, small linear size and proximity to the quasar, we can suppose that the absorber is formed by fragment(s) of planetary nebula(e) or AGB-star envelope(s) ejected into the intergalactic space by the quasar wind. Since gas and dust particles have different velocities in the stream, dust-gas separation occurs, and the gas carried away into the halo of the quasar host galaxy or even into the intergalactic space becomes dust-free. However, elements condensed into the dust remain deficient even in the dust-free absorber.

\subsubsection{System at $z_{a b s}=1.7529$}

The absorber at $z_{\mathrm{abs}}=1.7529$ exhibits a saturated H I $\lambda 1215$ line and strong lines of many carbon and silicon ions, all having very simple profile shapes (Fig. 3). The blue and red wings of the hydrogen line look different, and the metal lines are shifted relative to its center. Additionally, the flat bottom lines of the C IV $\lambda \lambda 1548,1550$ doublet and Si III $\lambda 1206$ show nonzero residual intensity at the line centers which means that the background radiation is leaking. The covering factor for these lines can be easily set as $C(\mathrm{C}$ IV $)=0.99, C(\mathrm{Si}$ III $)=0.98$. Clear profiles are also available for the doublets Si IV $\lambda \lambda 1393,1402$ and $\mathrm{Mg}$ II $\lambda \lambda 2796,2803$, so it is possible to estimate the accurate covering factors for these lines as well: $C(\mathrm{Si}$ IV $)=0.98$, and $C(\mathrm{Mg}$ II $)=0.98$. Assuming that ions $\mathrm{C}$ II and Si II trace the same gas as $\mathrm{Mg}$ II, we set $C(\mathrm{C}$ II $)=C($ Si II $)=0.98$.

The incomplete covering arises probably due to microlensing of the background quasar by some intervening galaxy. This naturally supposes a very small size of the absorber. In spite of the presence of only one saturated line of neutral hydrogen, it is possible to estimate its column density with a relatively high accuracy (up to 30\%) since the velocity dispersion of the gas can be well restricted by the numerous metal lines.

Given the ions of different ionization stages of the same element (C II, C IV, Si II, Si III, Si IV), the spectral shape of the ionizing background radiation can be restored rather accurately (see Fig. 4). In order to describe the observed intensities, $I_{\lambda}$, of all ions at the same value of $U_{0}$, the ionizing spectrum should have a soft slope with the index $\alpha=1.7-1.8$ between 1 and 4 Ryd, and a drop in the intensity at 4 Ryd by 3-4 times.

The expected position of the neutral oxygen line OI $\lambda 1302.16$ is blended with a strong forest absorption which prevents setting a limit on oxygen abundance. This unknown abundance represents an additional degree of freedom in the procedure of the spectral shape recovering: with scaled down (relative to carbon) oxygen abundance the above conditions are fulfilled for the spectral shapes which are softer at $E>4$ Ryd. An acceptable range for the ionizing spectra is shown in Fig. 4. In all cases the carbon content comes slightly oversolar (1.1-1.5 solar values). The physical parameters of the absorbing gas are given in Table 1, column densities and the element abundances - in Tables 2 and 3, respectively. 
Table 1. Physical parameters of metal-rich absorbers.

\begin{tabular}{cccccccccc}
\hline \hline$\#$ & QSO & $z_{\mathrm{em}}$ & $z_{\mathrm{abs}}$ & $\log U_{0}$ & $N(\mathrm{H}), \mathrm{cm}^{-2}$ & $n_{0}, \mathrm{~cm}^{-3}$ & $L, \mathrm{pc}$ & $\bar{T}_{4}^{\dagger}$ & Ref. $^{a}$ \\
\hline 1 & HE1347-2457 & 2.578 & 2.5745 & -2.3 & $3.5 \mathrm{E} 17$ & $>5 \mathrm{E}-3$ & $<25$ & 0.7 & 1 \\
2 & & & 1.7529 & -2.5 & $(5-8) \mathrm{E} 18$ & $0.007-0.1$ & $12-500$ & 1.1 & 1 \\
3 & & & 1.5080 & -2.5 & $(5-8) \mathrm{E} 18$ & $>7 \mathrm{E}-3$ & $<500$ & 1.1 & 1 \\
$4 A$ & HE0151-4326 & 2.775 & 2.4158 & $-2--1.5$ & $(3-7) \mathrm{E} 16$ & $>7 \mathrm{E}-4$ & $<30$ & $\sim 1.0$ & 1 \\
$4 B$ & & & 2.4158 & $-3.2--2.2$ & $(1-10) \mathrm{E} 18$ & $>(0.1-1) \mathrm{E}-2$ & $<300$ & $1.4-2.0$ & 1 \\
$4 C$ & & & 2.4158 & $-2--1.5$ & $(1.5-3.5) \mathrm{E} 16$ & $>7 \mathrm{E}-4$ & $<15$ & $\sim 1.0$ & 1 \\
5 & & & 1.7315 & -2.3 & $(5-6) \mathrm{E} 18$ & $>5 \mathrm{E}-3$ & $<300$ & 0.9 & 1 \\
6 & HE0001-2340 & 2.26 & 1.6514 & -2.6 & $(5-6) \mathrm{E} 17$ & $>8 \mathrm{E}-3$ & $<20$ & 0.8 & 1 \\
7 & & & 1.5770 & $-2.0--1.8$ & $(6-8) \mathrm{E} 16$ & $>1 \mathrm{E}-3$ & $<20$ & $\sim 0.2$ & 1 \\
8 & HE0141-3932 & \multirow{2}{*}{1.80} & 1.7817 & -2.5 & $(4-5) \mathrm{E} 17$ & $>7 \mathrm{E}-3$ & $<20$ & 1 & 2 \\
9 & HE2347-4342 & 2.88 & 2.8980 & $>-1.0$ & $\sim 1 \mathrm{E} 17$ & $>1 \mathrm{E}-4$ & $<300$ & 1 & 3 \\
10 & & & 1.7963 & -2.7 & $\sim 1 \mathrm{E} 17$ & $>2 \mathrm{E}-2$ & $<4$ & 0.6 & 4 \\
11 & Q0329-385 & 2.435 & 2.3520 & $\sim-0.75$ & $\sim 1 \mathrm{E} 17$ & $>1 \mathrm{E}-4$ & $<300$ & $2-3$ & 3 \\
\hline
\end{tabular}

${ }^{\dagger}$ Mean kinetic temperature in units of $10^{4} \mathrm{~K}$; ${ }^{a}$ references. - (1) present paper, (2) Reimers et al. 2005; (3) Levshakov et al. 2008; (4) Agafonova et al. (2007).

The abundance pattern is almost identical to that found in the previous $z_{\mathrm{abs}}=2.5745$ system: silicon is significantly depleted relative to carbon, magnesium and aluminium. The absolute abundance of magnesium depends on the depth of the break at 4 Ryd: more softer spectra give lower $\mathrm{Mg}$ content, so that the ratio $[\mathrm{Mg} / \mathrm{C}]$ can be by $\sim 0.1$ dex both below and above zero. A clear continuum window at the expected position of the Fe II $\lambda 2600$ line allows us to set an upper limit on the iron content: $[\mathrm{Fe} / \mathrm{C}]<-0.25$, i.e. iron is clearly underabundant relative to carbon. Unfortunately, both nitrogen lines N V $\lambda \lambda 1238.82,1242.80$ are contaminated by forest absorption, and only a not very instructive upper limit on the nitrogen content can be set, [N/C] $<0.2$.

The high metallicity and the relative ratios of $\mathrm{C}, \mathrm{Si}, \mathrm{Mg}, \mathrm{Al}$, with silicon and probably magnesium depleted compared to carbon and aluminium, suggest again (as it was for the previous $z_{\mathrm{abs}}=2.5745$ absorber) the dust-forming envelopes of the postmain sequence stars or planetary nebulae as a possible source of the observed absorption. The underabundance of iron relative to carbon can be explained by different reasons: it may be intrinsic due to a relatively low contribution from $\mathrm{SNe}$ Ia to the enrichment of gas, or it may be produced in the AGB-stars themselves through the depletion onto dust grains and/or $s$-process (Herwig 2005).

Clear and strong lines of $\mathrm{Mg}$ II make it possible to conduct one more test whether the AGB-stars are at play or not. Theory predicts that in the AGB-stars with masses $M>4 M_{\odot}$ the heavy magnesium isotopes ${ }^{25} \mathrm{Mg}$ and ${ }^{26} \mathrm{Mg}$ should be enhanced (Karakas \& Lattanzio 2003). Solar isotopic composition is ${ }^{24} \mathrm{Mg}:{ }^{25} \mathrm{Mg}:{ }^{26} \mathrm{Mg}=78.99: 10.00: 11.01$, but observations of red giant stars in globular clusters show in some cases much higher input of ${ }^{25} \mathrm{Mg}$ and ${ }^{26} \mathrm{Mg}$ (Yong et al. 2003, 2006). Due to secondary character of ${ }^{25} \mathrm{Mg}$ and ${ }^{26} \mathrm{Mg}$ the content of these isotopes is expected to scale with metallicity.

The wavelengths of the resonance transitions of $\mathrm{Mg}$ II are 2796.3553 $\AA$ for ${ }^{24} \mathrm{Mg}, 2796.3511 \AA$ for ${ }^{25} \mathrm{Mg}$, and $2796.3473 \AA$ for ${ }^{26} \mathrm{Mg}$ (Morton 2003). For solar isotopic composition Morton gives the weighted mean value $\lambda_{\text {rest }}=2796.3543 \AA$. If the abundances of the heavy isotopes are enhanced, then the wavelength $\lambda_{\text {rest }}$ becomes smaller, i.e. the observed at a given redshift $\mathrm{Mg}$ II lines are shifted toward lower radial velocities as compared with their positions expected for the solar isotopic composition ratio.

In our approach to calculate synthetic profiles we assume that all lines are produced by the same absorbing gas with fluctuating density and velocity fields. Profiles of specific ions may look different due to different responses of ions (ion fractions) to the local gas density which in the optically thin case are determined entirely by the spectral energy distribution of the ionizing background. This means that profiles - in spite of being different - should retain definite consistency governed by both the distributions of gas density and velocity along the line-of-sight and by the ionizing background. However, with magnesium lines centered using the solar composition wavelengths the synthetic profiles of $\mathrm{Mg}$ II and other low-ionization transitions C II $\lambda 1334$ and Si II $\lambda 1260$ look a bit incoherent (Fig. 5a).

On the contrary, when the observed profiles of $\mathrm{Mg}$ II are shifted by, e.g., $0.6 \mathrm{~km} \mathrm{~s}^{-1}$ towards higher radial velocities (which would correspond to the inversion of the solar ratio of ${ }^{24} \mathrm{Mg}$ to ${ }^{26} \mathrm{Mg}$, i.e. $\left.{ }^{24} \mathrm{Mg}:{ }^{25} \mathrm{Mg}:{ }^{26} \mathrm{Mg}=11: 10: 79\right)$, the quality of the fitting improves considerably (Fig. 5b).

However, with the available spectral data this test is to a certain extent qualitative: positive shift of magnesium lines is unambiguously preferred, but the accurate value of this shift cannot be determined since the fittings with $\mathrm{Mg}$ II lines shifted by $0.5 \mathrm{~km} \mathrm{~s}^{-1}$ and by $0.7 \mathrm{~km} \mathrm{~s}^{-1}$ are statistically indistinguishable. In fact, accurate measurements of the isotopic composition by means of a line shift require a specially observed and processed quasar spectrum (e.g., Levshakov et al. 2007; Molaro et al. 2008) and cannot be carried out with the present spectrum of HE13472457.

As already mentioned above, the linear size of the $z_{\mathrm{abs}}=$ 1.7529 system is probably very small. The upper limit (assuming the absorber is intergalactic) is $L<500 \mathrm{pc}$. The continuum window at the expected position of the $\mathrm{C} \mathrm{II}^{*} \lambda 1335$ line allows us to set an upper limit on its column density, $N\left(\mathrm{C} \mathrm{II}^{*}\right) \lesssim 9.0 \times 10^{11}$ $\mathrm{cm}^{-2}$ which results in an upper limit on the gas density of $n_{\mathrm{H}} \lesssim 0.14 \mathrm{~cm}^{-3}$ (see Sect. 2.2.1 in Paper I for details of calculations). With the total hydrogen column density of $(5-10) \times$ $10^{18} \mathrm{~cm}^{-2}$ this gives $L \gtrsim 12 \mathrm{pc}$.

\subsubsection{System at $z_{\mathrm{abs}}=1.5080$}

This system exhibits one saturated and very noisy hydrogen line (lies at the edge of observational wavelength range) accompanied by strong lines of metal ions in different ionization stages (Fig. 6). Clear central parts of the doublets C IV $\lambda 1548,1550$ and Si IV $\lambda 1393,1402$ allow us to calculate accurately the corresponding covering factors: $C(\mathrm{C}$ IV $)=C(\mathrm{Si}$ IV $)=0.99$. The same covering factors were adopted for all other lines. Given only one pair of the subsequent ions, Si II and Si IV (C II $\lambda 1334$ is blended 
Table 2. Calculated column densities for atoms and ions identified in metal-rich absorbers (for references, see Table 1).

\begin{tabular}{|c|c|c|c|c|c|c|}
\hline $\begin{array}{c}\text { Redshift, } \\
z_{\text {abs }}\end{array}$ & $\overline{\mathrm{HI}}$ & $\begin{array}{l}\text { C II } \\
\text { C III } \\
\text { C IV } \\
\end{array}$ & $\begin{array}{l}\text { N II } \\
\text { N III } \\
\text { N V } \\
\end{array}$ & $\begin{array}{l}\text { O I } \\
\text { O VI }\end{array}$ & $\begin{array}{l}\mathrm{Mg} \text { II } \\
\text { Fe II } \\
\text { Al III } \\
\end{array}$ & $\begin{array}{l}\text { Si II } \\
\text { Si III } \\
\text { Si IV } \\
\end{array}$ \\
\hline \multicolumn{7}{|c|}{$H E 1347-2457\left(z_{\mathrm{em}}=2.578\right)$} \\
\hline $2.5745^{*}$ & $(9.5 \pm 0.3) \mathrm{E} 14$ & $\begin{array}{l}(1.6 \pm 0.2) \mathrm{E} 13 \\
(1.9 \pm 0.3) \mathrm{E} 14 \\
(3.2 \pm 0.1) \mathrm{E} 13\end{array}$ & $\begin{array}{c}(1.8 \pm \overline{0} .2) \mathrm{E} 13 \\
<5.0 \mathrm{E} 11\end{array}$ & - & $\begin{array}{l}(1.3 \pm 0.2) \mathrm{E} 12 \\
(3.6 \pm 0.5) \mathrm{E} 11\end{array}$ & $\begin{array}{l}(1.4 \pm 0.2) \mathrm{E} 12 \\
(4.7 \pm 0.4) \mathrm{E} 12 \\
(3.7 \pm 0.3) \mathrm{E} 13\end{array}$ \\
\hline $1.7529 *$ & $(1.0-1.5) \mathrm{E} 16$ & $(1.5 \pm \underset{-}{0.1) \mathrm{E} 14}$ & $\begin{array}{l}- \\
-\end{array}$ & - & $\begin{array}{c}(6.8 \pm 0.2) \mathrm{E} 12 \\
<3.5 \mathrm{E} 11\end{array}$ & $\begin{array}{l}(9.2 \pm 1.0) \mathrm{E} 12 \\
(5.5 \pm 0.5) \mathrm{E} 13\end{array}$ \\
\hline $1.5080^{*}$ & $(1.0-1.5) \mathrm{E} 16$ & $\begin{array}{c}(3.2 \pm 0.5) \mathrm{E} 14 \\
- \\
(1.7 \pm 0 . \\
0.2) \mathrm{E} 14\end{array}$ & $\begin{array}{c}<1.0 \mathrm{E} 13 \\
- \\
<7 . \overline{0} \mathrm{E} 12\end{array}$ & $\begin{array}{l}- \\
-\end{array}$ & $\begin{array}{l}(1.7 \pm 0.2) \mathrm{E} 12 \\
(1.1 \pm 0.1) \mathrm{E} 13 \\
(2.1 \pm 0.2) \mathrm{E} 12 \\
(2.2 \pm 0.2) \mathrm{E} 12\end{array}$ & $\begin{array}{c}(4.5 \pm 0.5) \mathrm{E} 13 \\
(1.5 \pm 0.1) \mathrm{E} 13 \\
(5.4 \pm 0.5) \mathrm{E} 13\end{array}$ \\
\hline \multicolumn{7}{|c|}{$H E 0151-4326\left(z_{\mathrm{em}}=2.775\right)$} \\
\hline $\begin{array}{c}2.4158^{\diamond} \\
A\end{array}$ & $(2.2 \pm 0.1) \mathrm{E} 13$ & $\begin{array}{c}<5.0 \mathrm{E} 11 \\
(1.5 \pm 0.2) \mathrm{E} 13 \\
(1.2 \pm 0.1) \mathrm{E} 13\end{array}$ & $<1 . \overline{1} \mathrm{E} 13$ & $\begin{array}{l}- \\
-\end{array}$ & $\begin{array}{l}- \\
- \\
-\end{array}$ & $\begin{array}{l}<8 . \overline{5} \mathrm{E} 10 \\
<2.5 \mathrm{E} 11\end{array}$ \\
\hline$B$ & $(7.5 \pm 0.5) \mathrm{E} 15$ & $\begin{array}{c}<1.0 \mathrm{E} 12 \\
(6.5 \pm 1.0) \mathrm{E} 12 \\
<1.0 \mathrm{E} 12\end{array}$ & $\begin{array}{c}<4.0 \mathrm{E} 12 \\
\leq 7.0 \mathrm{E} 12 \\
-\end{array}$ & - & $\begin{array}{l}- \\
- \\
-\end{array}$ & $\begin{array}{l}<2 . \overline{8 \mathrm{E}} 11 \\
<2.5 \mathrm{E} 11\end{array}$ \\
\hline$C$ & $(1.0 \pm 0.2) \mathrm{E} 13$ & $\begin{array}{l}(6.5 \pm \overline{0} .5) \mathrm{E} 12 \\
(7.0 \pm 0.5) \mathrm{E} 12\end{array}$ & $\begin{array}{c}\ddagger \\
(5-6) \mathrm{E} 12 \\
-\end{array}$ & $<4 . \overline{5} \mathrm{E} 12$ & $\begin{array}{l}- \\
- \\
-\end{array}$ & $\begin{array}{c}- \\
<2 . \overline{\mathrm{E}} 11\end{array}$ \\
\hline 1.7315 & $(0.9-1.2) \mathrm{E} 16$ & $\begin{array}{l}(1.0 \pm 0.1) \mathrm{E} 14 \\
(3.2 \pm 0.5) \mathrm{E} 14\end{array}$ & $\begin{array}{c}- \\
<1 . \overline{5} \mathrm{E} 13\end{array}$ & $\begin{array}{l}- \\
-\end{array}$ & $\begin{array}{l}(9.0 \pm 1.0) \mathrm{E} 12 \\
(3.4 \pm 0.3) \mathrm{E} 11 \\
(1.3 \pm 0.2) \mathrm{E} 12\end{array}$ & $\begin{array}{l}(1.0 \pm 0.1) \mathrm{E} 13 \\
(3.6 \pm 0.5) \mathrm{E} 13 \\
(3.8 \pm 0.5) \mathrm{E} 13\end{array}$ \\
\hline \multicolumn{7}{|c|}{$H E 0001-2340\left(z_{\mathrm{em}}=2.26\right)$} \\
\hline 1.6514 & $(3.5-4.5) \mathrm{E} 15$ & $\begin{array}{c}\$ 3.7 \mathrm{E} 13 \\
(4.7 \pm \overline{0} .2) \mathrm{E} 13\end{array}$ & $\begin{array}{l}- \\
- \\
-\end{array}$ & $\begin{array}{c}<1.0 \mathrm{E} 12 \\
-\end{array}$ & $\begin{array}{c}(4.1 \pm 0.4) \mathrm{E} 12 \\
(2.0 \pm 0.2) \mathrm{E} 11 \\
<1.3 \mathrm{E} 11\end{array}$ & $\begin{array}{l}(1.8 \pm 0.2) \mathrm{E} 12 \\
(2.5 \pm 0.4) \mathrm{E} 12 \\
(2.2 \pm 0.2) \mathrm{E} 12\end{array}$ \\
\hline 1.5770 & $(0.9-1.0) \mathrm{E} 14$ & $\begin{array}{c}(7.6 \pm 0.7) \mathrm{E} 12 \\
(5.2 \pm 0.5) \mathrm{D} 13\end{array}$ & $\begin{array}{c}- \\
\stackrel{-}{\ddagger}(0.8-1.1) \mathrm{E} 13\end{array}$ & $\begin{array}{l}- \\
-\end{array}$ & $\begin{array}{l}<3.0 \mathrm{E} 11 \\
<1 . \overline{3} \mathrm{E} 11\end{array}$ & 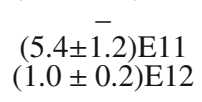 \\
\hline 1.7817 & $(2.5-3.0) \mathrm{E} 15$ & $\begin{array}{c}H \\
(1.64 \pm 0.08) \mathrm{E} 13 \\
(2.2 \pm 0.2) \mathrm{E} 13\end{array}$ & $\begin{array}{c}0141-3932\left(z_{\mathrm{em}}\right. \\
- \\
<1 . \overline{\mathrm{E}} 12\end{array}$ & 1.80) & $\begin{array}{l}(1.2 \pm 0.2) \mathrm{E} 12 \\
(1.4 \pm 0.4) \mathrm{E} 11 \\
(3.6 \pm 1.0) \mathrm{E} 11\end{array}$ & $\begin{array}{c}(2.8 \pm 0.3) \mathrm{E} 12 \\
(4.5 \pm 0.4) \mathrm{E} 12\end{array}$ \\
\hline \multicolumn{7}{|c|}{$H E 2347-4342\left(z_{\mathrm{em}}=2.88\right)$} \\
\hline $2.8980^{*}$ & $(1.1-2.3) \mathrm{E} 13$ & $\begin{array}{c}<3.0 \mathrm{E} 11 \\
(5-9) \mathrm{E} 12 \\
(1.2 \pm 0.1) \mathrm{E} 14\end{array}$ & $\begin{array}{c}- \\
(2.6 \pm \overline{0} .2) \mathrm{E} 13\end{array}$ & $(2.8 \pm \overline{0} .2) \mathrm{E} 14$ & $\begin{array}{l}- \\
-\end{array}$ & $\begin{array}{l}- \\
- \\
-\end{array}$ \\
\hline 1.7963 & $(0.9-1.2) \mathrm{E} 16$ & 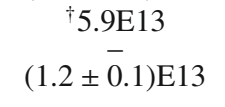 & $\begin{array}{l}- \\
- \\
-\end{array}$ & $\begin{array}{c}(1.0 \pm \underset{-}{0.2) \mathrm{E} 13} \\
\end{array}$ & $\begin{array}{l}(0.9-1.6) \mathrm{E} 13 \\
(9.4 \pm 0.5) \mathrm{E} 11 \\
(7.4 \pm 0.7) \mathrm{E} 11\end{array}$ & $\begin{array}{l}(1.4 \pm 0.1) \mathrm{E} 13 \\
(4.9 \pm 1.0) \mathrm{E} 12 \\
(2.9 \pm 0.3) \mathrm{E} 12\end{array}$ \\
\hline 2.3520 & $(0.7-1.3) \mathrm{E} 13$ & $\begin{array}{c}<5.0 \mathrm{E} 11 \\
\dagger 3.6 \mathrm{E} 12 \\
(3.2 \pm 0.1) \mathrm{E} 13\end{array}$ & $\begin{array}{c}329-385\left(z_{\mathrm{em}}=\right. \\
- \\
\dagger 2.9 \mathrm{E} 12 \\
(4.0 \pm 0.2) \mathrm{E} 13\end{array}$ & $\begin{array}{l}\text { 435) } \\
(1.2 \pm 0.1) \mathrm{E} 14\end{array}$ & $\begin{array}{l}- \\
- \\
-\end{array}$ & $\begin{array}{l}- \\
- \\
-\end{array}$ \\
\hline
\end{tabular}

${ }^{*}$ Incomplete covering of the background light source; ${ }^{\circ} A:-40<v<30 \mathrm{~km} \mathrm{~s}^{-1}, B: 130<v<330 \mathrm{~km} \mathrm{~s}^{-1}, C: 300<v<370 \mathrm{~km} \mathrm{~s}{ }^{-1}$, see Fig. 8 ;

${ }^{\ddagger}$ deconvolved from Ly $-\alpha$ forest absorption; ${ }^{\dagger}$ calculated with the recovered velocity and density distributions.

with a deep forest absorption and cannot be deconvolved) and unknown relative element abundances, it is impossible to conclude about the SED of the ionizing radiation. However, the strong C IV and the pronounced Fe II lines point to a spectrum at least as hard at $E>4$ Ryd as that estimated from the previous $z_{\text {abs }}=1.7529$ system (Fig. 4) - otherwise an overabundance of iron to carbon significantly exceeds solar value, $[\mathrm{Fe} / \mathrm{C}] \gtrsim 1.0$.

For the same reason spectra with the indices $\alpha \sim 1.5-1.8$ between 1 and 4 Ryd are preferable. With such spectra, abundances of carbon and silicon come out to be slightly oversolar, however without deficit of silicon (cf. with column densities in the $z_{\mathrm{abs}}=$ 1.7529 system): $[\mathrm{C} / \mathrm{H}] \sim[\mathrm{Si} / \mathrm{H}] \sim 0.1-0.2$, whereas magnesium, aluminium and iron are strongly enhanced relative to carbon: $[\mathrm{Mg} / \mathrm{C}]>0.2,[\mathrm{Al} / \mathrm{C}]>0.15,[\mathrm{Fe} / \mathrm{C}]>0.3$, with very probable overabundance of iron to magnesium as well, $[\mathrm{Fe} / \mathrm{Mg}]>0.1$.
There is a continuum window at the expected position of the $\mathrm{N} \mathrm{V}$ $\lambda 1238$ line which provides a conservative upper limit on the nitrogen abundance $[\mathrm{N} / \mathrm{C}]<0.3$. The physical parameters, column densities and derived abundances are given in Tables 1-3. Very similar abundances were previously obtained for the narrow-line associated system at $z_{\mathrm{abs}}=1.7817$ towards HE0141-3932 (\# 8 in Tables 2 and 3, see also Sect. 4.2 in Reimers et al. 2005). Such pattern - with iron enhanced relative to $\alpha$-elements - is often observed in giants in local dwarf galaxies (Venn et al. 2004; Bonifacio et al. 2004; Tautvaišienė et al. 2007) and was also registered in some metal-rich giant stars in the Milky Way (Fabbian et al. 2005).

As it was the case for the $z_{\mathrm{abs}}=1.7529$ system (Sect. 3.1.2), simultaneous fitting of all lines in the present system results in systematically shifted low-ionization lines when $\mathrm{Mg}$ II is 
Table 3. Abundance patters for the absorbers listed in Table 1. $[\mathrm{X} / \mathrm{Y}]$ means $\log \left(N_{\mathrm{X}} / N_{\mathrm{Y}}\right)-\log \left(N_{\mathrm{X}} / N_{\mathrm{Y}}\right)_{\odot}$. Point estimates have uncertainties of 0.05 dex.

\begin{tabular}{ccccccccc}
\hline \hline$\#$ & $z_{\text {abs }}$ & {$[\mathrm{C} / \mathrm{H}]$} & {$[\mathrm{N} / \mathrm{C}]$} & {$[\mathrm{O} / \mathrm{C}]$} & {$[\mathrm{Mg} / \mathrm{C}]$} & {$[\mathrm{Al} / \mathrm{C}]$} & {$[\mathrm{Si} / \mathrm{C}]$} & {$[\mathrm{Fe} / \mathrm{C}]$} \\
\hline 1 & 2.5745 & $0.4-0.5$ & -0.2 & - & $0-0.1$ & 0.0 & -0.3 & - \\
2 & 1.7529 & $0.1-0.2$ & $<0.2$ & - & $-0.1-0.1$ & 0.0 & -0.3 & $<-0.25$ \\
3 & 1.5080 & $0.1-0.2$ & $<0.3$ & - & $\gtrsim 0.2$ & $\gtrsim 0.15$ & 0.0 & $\gtrsim 0.3$ \\
$4 A$ & 2.4158 & $0.2-0.5$ & $<0.5$ & - & - & - & $<-0.6$ & - \\
$4 B$ & 2.4158 & $-1.5--2.5$ & $<0.5$ & - & - & - & $<-0.5$ & - \\
$4 C$ & 2.4158 & $0.2-0.3$ & $<0.5$ & - & - & - & $<-0.6$ & - \\
5 & 1.7315 & $0.2-0.35$ & $<0.1$ & - & 0.25 & 0.0 & -0.25 & -0.2 \\
6 & 1.6514 & $0.40-0.45$ & - & $<0.1$ & $0.1-0.15$ & $<-0.6$ & -0.8 & $\sim-0.3$ \\
7 & 1.5770 & $\sim 1.0$ & $0.3-0.5$ & - & $<0$ & $<0$ & $-1.0--0.7$ & - \\
8 & 1.7817 & 0.15 & $<0.0$ & - & 0.15 & 0.15 & -0.05 & $0.15-0.25$ \\
9 & 2.8980 & $\sim 1.0$ & -0.3 & $0.0-0.5$ & - & - & - & - \\
10 & 1.7963 & 0.5 & - & 0.0 & $0.2-0.4$ & 0.12 & 0.0 & -0.55 \\
11 & 2.3520 & $0.3-0.5$ & 0.4 & 0.0 & - & - & - & - \\
\hline
\end{tabular}

centered using the solar isotopic ratio (Fig. 7a), whereas $\mathrm{Mg}$ II lines shifted by $\sim 0.6 \mathrm{~km} \mathrm{~s}^{-1}$ redward produce almost perfect fitting (Fig. 7b). Thus, the enhanced content of heavy Mg isotopes seems very probable which means that AGB-stars contributed to the metal enrichment of gas in the $z_{\mathrm{abs}}=1.5080$ system.

\subsection{Quasar HE0151-4326}

\subsubsection{System at $z=2.4158$}

The absorbing complex at $z_{\mathrm{abs}}=2.4158$ was firstly mentioned in Aracil et al. (2004). It is spread over $350 \mathrm{~km} \mathrm{~s}^{-1}$ and can be divided into three parts (Fig. 8): two systems with weak H I Ly- $\alpha$ lines and pronounced metal lines of C III $\lambda 977$ and C IV $\lambda \lambda 1548,1550$ centered at $v=0 \mathrm{~km} \mathrm{~s}^{-1}$ (subsystem $A$ ) and $v=347 \mathrm{~km} \mathrm{~s}^{-1}$ (subsystem $C, z_{\mathrm{abs}}=2.4196$ ), and a subsystem $B$ comprising a strong $\mathrm{HI}$ absorption in the range $140<v<$ $340 \mathrm{~km} \mathrm{~s}^{-1}\left(z_{\mathrm{abs}}=2.4180\right)$ accompanied by only one prominent metal line - C III $\lambda$ 977. That the observed absorptions are due to $\mathrm{C} \mathrm{III} \mathrm{is} \mathrm{quite} \mathrm{certain:} \mathrm{lines} \mathrm{are} \mathrm{narrow} \mathrm{(i.e.} \mathrm{cannot} \mathrm{be} \mathrm{hydrogen}$ lines from the Ly- $\alpha$ forest), aligned in velocity with the hydrogen absorption, and there are no any plausible metal contaminants from the identified intervening systems.

In the subsystems $B$ and $C$, there are weak and narrow lines at the expected positions of the N III $\lambda 989$ (in $C$ - blended with broad and shallow $\mathrm{H}$ I forest absorption). No candidate for blending from other systems was found, so quite probably these weak absorptions are indeed due to N III $\lambda 289$. From the N V doublet, only a small unblended part of the $\mathrm{N} V \lambda 1238$ line is present in the subsystem $A$. The $\mathrm{O}$ VI doublet has an unblended part in the O VI $\lambda 1037$ line seen in the subsystem $C$.

The C II $\lambda 1334$ lines coincide with a broad and shallow intrinsic quasar absorption of H I Ly- $\alpha$ (HE0151-4326 is a miniBAL quasar), but the absence of any pronounced features at the expected positions of the $\mathrm{C}$ II lines in all three subsystems leads to the conclusion that these lines should be very weak. Continuum windows at the positions of the silicon lines Si III $\lambda 1206$ and Si IV $\lambda 1393$ give upper limits on their column densities. Unfortunately, both lines of $\mathrm{Mg}$ II $\lambda 2796,2803$ coincide with strong telluric absorptions.

With ions available in all three sybsystems it is not possible to restore the spectral shape of the underlying ionizing background uniquely: the observed line profiles can be described with UVB spectra ranging from pure power laws to power laws with intensity break of different depths at 4 Ryd. The blue wing of the H I Ly- $\alpha$ line in the subsystem $A$ turned out to be inconsistent with the assumption of constant metallicity throughout the absorber. The blue wing of the synthetic Ly- $\alpha$ profile was then calculated on base of density and velocity distributions restored from metal ions and the red part of the H I Ly- $\alpha$ assuming a constant metal content of the absorbing gas.

The column densities of all ions are listed in Table 2. The subsystems $A$ and $C$ reveal almost identical ratios C IV/C III $\sim 1$ and $\mathrm{C} \mathrm{IV} / \mathrm{H} \mathrm{I} \sim 0.5$ indicating that these absorbers have very similar ionization states and metallicities. In contrast, the absorber $B$ with its ratio $\mathrm{C}$ IV $/ \mathrm{C} \mathrm{III}<0.15$ and more than two orders of magnitude higher column density of neutral hydrogen, $N(\mathrm{HI})=$ $7.5 \times 10^{15} \mathrm{~cm}^{-2}$, seems to be different both in physical state of the gas, and in metal content.

In spite of the uncertainty in the UVB shape, element abundances retain a remarkably stable pattern (Table 3 ). In particular, in the subsystems $A$ and $C$ at the value of the ionization parameter $U$ corresponding to $\Upsilon_{\mathrm{C}_{\text {IV }}} / \Upsilon_{\mathrm{C}}$ III $\sim 1 \Upsilon \Upsilon_{i}$ is a fraction of ion $i$ ) all ionizing spectra give oversolar abundance of carbon which can reach a few solar values (the harder at $E>4$ Ryd spectrum the higher carbon content). On the other hand, the fractions of silicon ions Si III and Si IV at $U$ corresponding to $\Upsilon_{\mathrm{C} \text { IV }} / \Upsilon_{\mathrm{C}_{\text {III }}} \sim 1$ are such high that only a significantly underabundant silicon can comply with upper limits on $N($ Si III) and $N(\mathrm{Si}$ IV $):[\mathrm{Si} / \mathrm{C}]<-0.6$. At this $U$ for all spectra we also have $\Upsilon_{\mathrm{C} \text { III }}=\Upsilon_{\mathrm{N} \text { III }}$. If the line in the subsystem $C$ is indeed due to N III $\lambda 989$ absorption, then we obtain a clear overabundance of nitrogen to carbon, $[\mathrm{N} / \mathrm{C}] \sim 0.5$.

Unfortunately, the upper limit on $N(\mathrm{O}$ VI) from the subsystem $C$ cannot be translated into a reasonable bound on the oxygen abundance: the $\mathrm{O}$ VI fraction is extremely sensitive to the energy level above 4 Ryd, but since this level cannot be estimated from the available ions, variations of the oxygen abundance for applicable ionizing spectra exceed an order of magnitude.

In the subsystem $B$, the available limits $\mathrm{C}$ IV $/ \mathrm{C}$ III $<0.15$ and $\mathrm{N}$ II $/ \mathrm{N}$ III $<0.6$ provide bounds to the acceptable $U$ range: $-3.2<\log (U)<-2.25$. In this $U$ range, $\log \left(\Upsilon_{\mathrm{HI}}\right) \sim-2.2-$ -3.2 , the fractions of $\mathrm{C}$ III and N III remain almost constant, $\log \left(\Upsilon_{\mathrm{C} \text { III }}\right) \approx \log \left(\Upsilon_{\mathrm{N}_{\text {III }}}\right) \sim-0.05$, and the fraction of Si III changes from -0.1 to -0.2 . All values depend only weakly on the spectral shape. Thus, the measured column densities lead to the following abundances: $[\mathrm{C} / \mathrm{H}] \sim-2.5--1.5,[\mathrm{Si} / \mathrm{C}]<-0.5$, and $[\mathrm{N} / \mathrm{C}]$ $\sim 0.5-0.6$ (providing that N III $\lambda 989$ absorption in subsystem $B$ is real). 

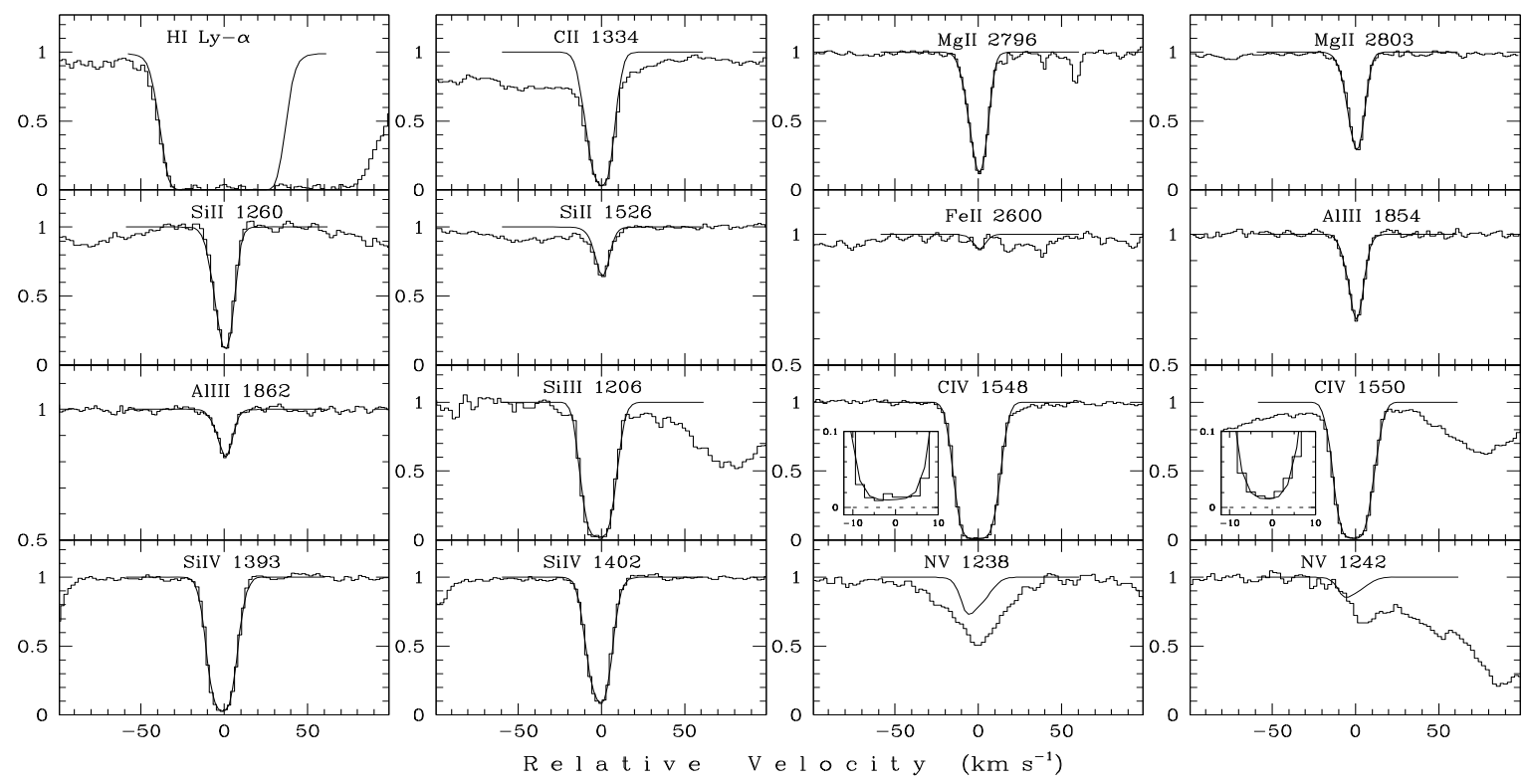

Fig. 3. Same as Fig. 1 but for the $z_{\mathrm{abs}}=1.7529$ system (HE1347-2457). The vertical axis is normalized intensities. The zero radial velocity is fixed at $z=1.7529$.

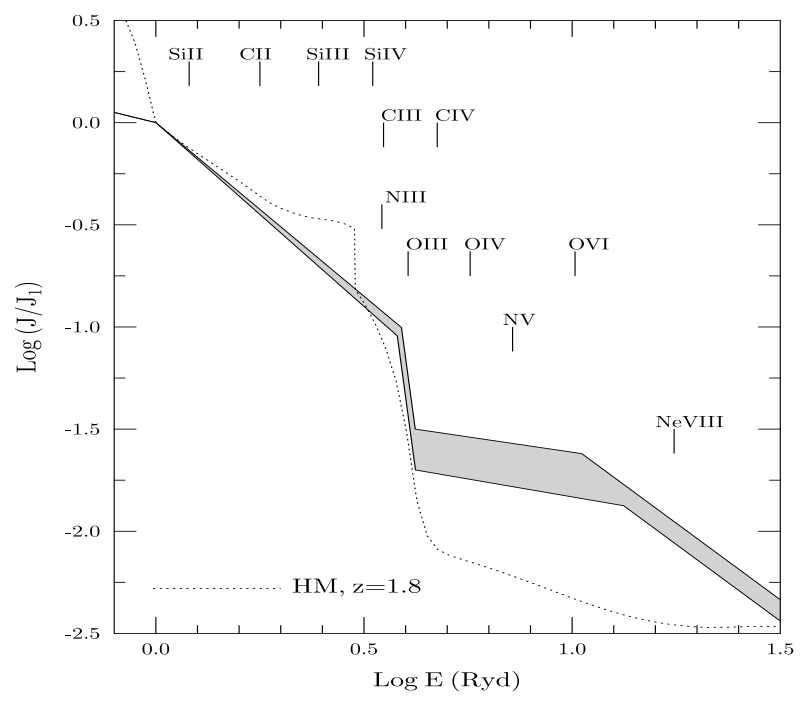

Fig. 4. Same as Fig. 2 but for the $z_{\text {abs }}=1.7529$ system (HE1347-2457).

This kind of the abundance pattern when a low carbon content $(1 / 300$ to $1 / 30$ solar) is accompanied by a strong underabundance of silicon (and probably by 3-times overabundance of nitrogen) is highly unusual for a stand-alone intergalactic absorber with a comparable neutral hydrogen column density, $N(\mathrm{HI}) \sim 10^{16} \mathrm{~cm}^{-2}$, but it coincides with [Si/C] (and [N/C]) measured in the subsystems $A$ and $C$. Such a similarity points to a physical connection between all three absorbers. Note that the ionization parameter $U_{0}$ in the metal-poor subsystem $B$ is signifucantly lower than that in the metal-rich subsystems $A$ and $C$, i.e. the gas density in the subsystem $B$ is higher (the kinetic temperature is higher as well because of low metallicity).

Absorption systems with strong metal lines and low column densities of neutral hydrogen, $N(\mathrm{HI})<10^{14} \mathrm{~cm}^{-2}$, such as the subsystems $A$ and $C$, are known from the literature (e.g., Schaye et al. 2007). For these systems the derived metal abundances may be artificially boosted because of a possible overionization of H I (see, e.g., Sect. 2.3.1 in Paper I). However, the

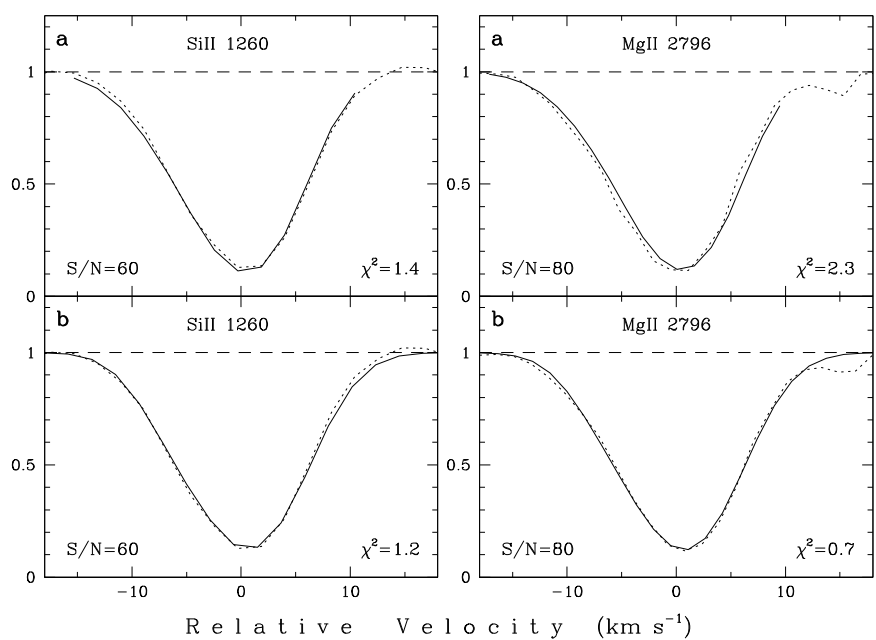

Fig. 5. $\mathrm{Mg}$ II in the $z_{\mathrm{abs}}=1.7529$ system $($ HE1347-2457). Panel a): observed (points) and synthetic (solid lines) profiles when $\mathrm{Mg}$ II lines are centered using the solar composition of the isotopes ${ }^{24} \mathrm{Mg}:{ }^{25} \mathrm{Mg}:{ }^{26} \mathrm{Mg}=79: 10: 11$. Panel b): observed (points) and synthetic (solid lines) profiles when $\mathrm{Mg}$ II lines are shifted by $0.6 \mathrm{~km} \mathrm{~s}^{-1}$ which corresponds to the inverse isotope composition ${ }^{24} \mathrm{Mg}:{ }^{25} \mathrm{Mg}:{ }^{26} \mathrm{Mg}=11: 10: 79$. The corresponding signal-to-noise $(\mathrm{S} / \mathrm{N})$ mean value per resolution element at the continuum level and the obtained $\chi_{\min }^{2}$ per degree of freedom are shown.

upper limit on $N(\mathrm{O} \mathrm{VI})<4.5 \times 10^{12} \mathrm{~cm}^{-2}$ and the low value of the ratio $N(\mathrm{O}$ VI $) / N(\mathrm{C}$ IV $)<0.65$ in the subsystem $C$ show that gas is close to the ionization equilibrium. Thus, the measured high metal abundances are probably real.

The obtained abundance pattern with silicon deficient (and nitrogen overabundant) to carbon, resembles patterns (taking silicon as a proxy to oxygen) observed in planetary nebulae in the Milky Way as well as in the LMC and SMC (Stanghellini 2007). Carbon (and nitrogen) are obviously enhanced due to dredge-up processes in the progenitor star(s). Whether a low content of silicon is intrinsic or affected by some depletion into dust is not clear since there are no other elements available. 

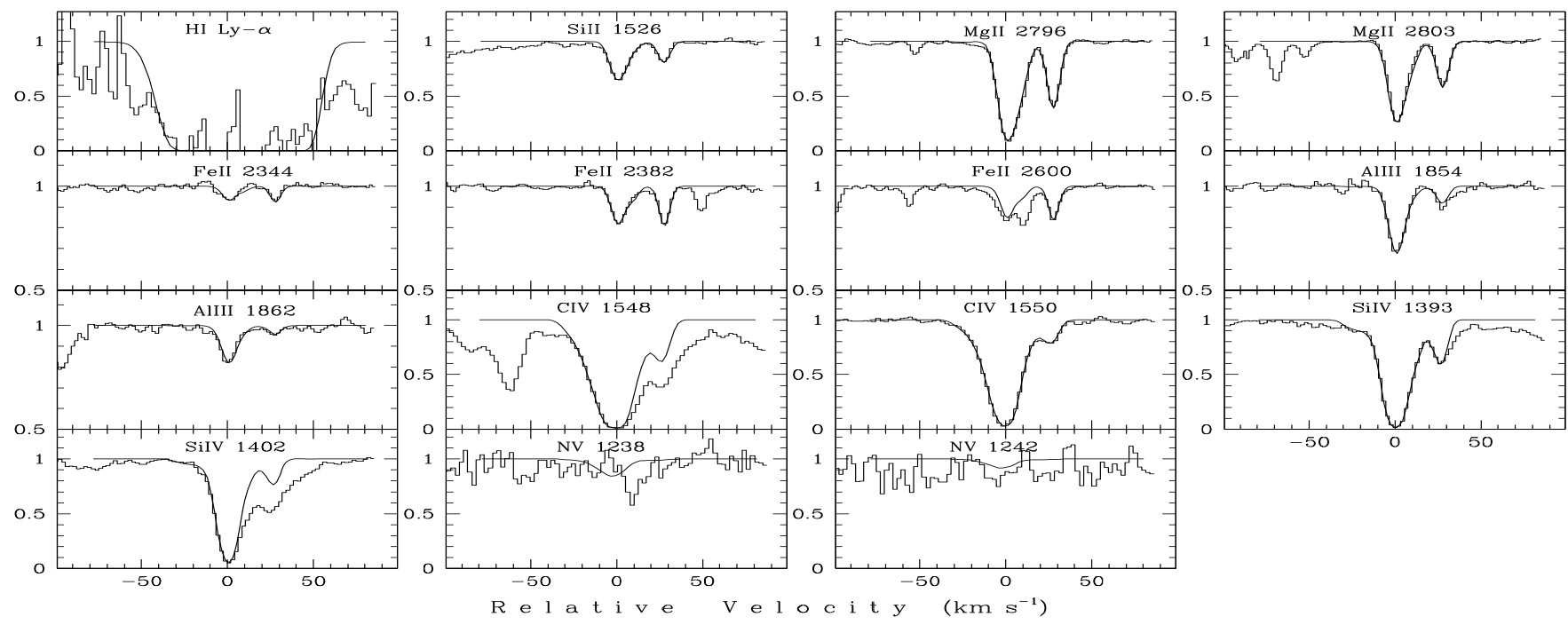

Fig. 6. Same as Fig. 1 but for the $z_{\text {abs }}=1.5080$ system (HE1347-2457).

It is known that many planetary nebulae/AGB-stars have cold outer envelopes (see, e.g., Marengo 2009, and references therein). In order to explain systematically higher abundances derived from weak optical recombination lines as compared to the abundances resulting from collisionally excited lines, a model of planetary nebula (PN) assuming the presence of $\mathrm{H}$ deficient (i.e. metal-rich) inclusions embedded in the diffuse nebular gas is discussed (e.g., Middlemass 1990; Liu et al. 2004; Wesson \& Liu 2004; Zhang et al. 2005). Accounting for this model, the $z_{\mathrm{abs}}=2.4158$ absorption system can be interpreted as $\mathrm{PN}$ fragment(s) transported into the IGM by the AGN/galactic wind.

In Paper I, we described a system at $z_{\mathrm{abs}}=2.3520$ towards Q0329-385 with an oversolar carbon content and overabundance of nitrogen to carbon (Table 3 ). This system exhibits a weak H I Ly- $\alpha$ accompanied by strong metal lines (C IV, N V, $\mathrm{O}$ VI), i.e. resembles the subsystems $A$ and $C$, but is much higher ionized. The $z_{\mathrm{abs}}=2.3520$ system is shifted by $\sim 7400 \mathrm{~km} \mathrm{~s}^{-1}$ from Q0329-385 and was classified in Paper I as a probable eject from the quasar host galaxy. While analysing this system, we have not considered the nearby absorption systems. However, detached by only $230 \mathrm{~km} \mathrm{~s}^{-1}\left(z_{\mathrm{abs}}=2.3545\right)$ there is an absorber with properties similar to those of the subsystem $B$ : strong $\mathrm{H}$ I Ly- $\alpha$ along with clear continuum at the positions of the C II, C IV, Si II, Si IV, and N V lines (Fig. 9). Weak absorptions are present at the positions of C III $\lambda 977$ and O VI $\lambda 1032$, but since both fall in the Ly- $\alpha$ forest, the observed intensities are only upper limits for these lines. The column density of H I can be estimated quite accurately (Ly- $\beta$ and Ly- $\gamma$ available): $N(\mathrm{HI})=$ $(4.5 \pm 0.3) \times 10^{14} \mathrm{~cm}^{-2}$. With such a high column density Ly$\alpha$ should be fully saturated - but it shows a flat bottom with the residual intensity of 0.025 (see insert in Fig. 9). This means that the absorber is small and does not completely cover the light source. At the same time, the system at $z_{\mathrm{abs}}=2.3520$ with $N(\mathrm{H} \mathrm{I})$ $\sim 10^{13} \mathrm{~cm}^{-2}$, the total hydrogen column $N(\mathrm{H})$ of a few units of $10^{17} \mathrm{~cm}^{-2}$ and the gas density $n>10^{-4} \mathrm{~cm}^{-3}$ (Table 1) does not show incomplete coverage, i.e. in any case it is not smaller than the system at $z_{\mathrm{abs}}=2.3545$. Therefore, the absorber with $N(\mathrm{H} \mathrm{I})=4.5 \times 10^{14} \mathrm{~cm}^{-2}$ should have $N(\mathrm{H})<10^{18} \mathrm{~cm}^{-2}$ and $n>10^{-3} \mathrm{~cm}^{-3}$ which is realized at $\log U<-2$, i.e. it cannot be a highly ionized system (and apparent absorption at the

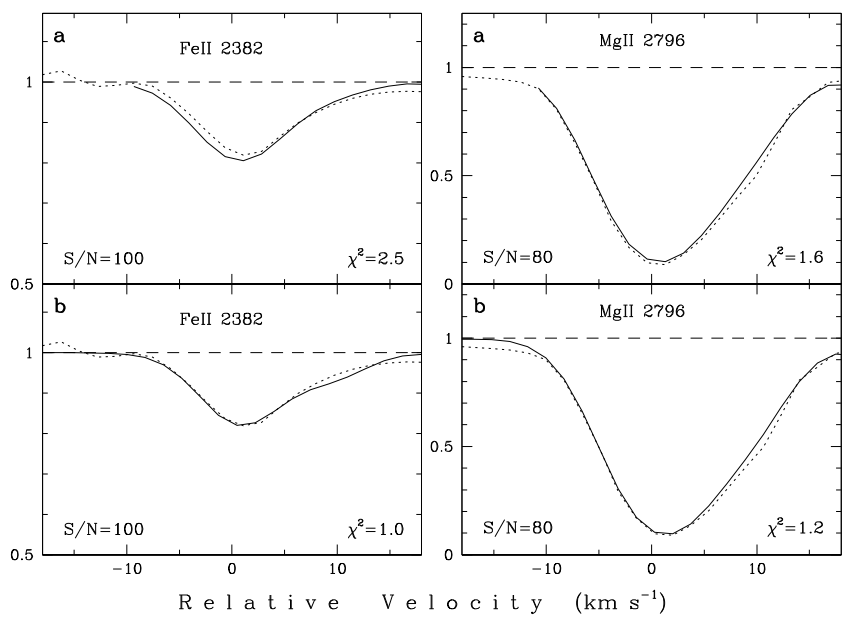

Fig. 7. Same as Fig. 5 but for the $z_{\mathrm{abs}}=1.5080$ system (HE1347-2457). In contrast to Fig. 5a where both lines $\mathrm{Si}$ II and $\mathrm{Mg}$ II are equivalently strong, here Fe II is significantly weaker than $\mathrm{Mg}$ II and, hence, the weight of $\mathrm{Mg}$ II dominates in the minimization of the objective function leading to a prominent shift of the Fe II line.

position of O VI $\lambda 1032$ is obviously due to some forest line). With the upper limit on $N(\mathrm{C}$ III $)<3.6 \times 10^{12} \mathrm{~cm}^{-2}$ we obtain for the carbon content the estimate $[\mathrm{C} / \mathrm{H}]<-1.0$. Unfortunately, positions of Si III $\lambda 1206$ and N III $\lambda 989$ are blended and any conclusive estimates on silicon and nitrogen contents are impossible. Thus, the system at $z_{\mathrm{abs}}=2.3545$ comprises low-ionization and metal-poor gas - as was the case for the subsystem $B$, whereas the $z_{\mathrm{abs}}=2.3520$ absorber - just as the subsystems $A$ and $C-$ is formed by metal-rich and highly ionized gas. Surprisingly, even the velocity offset between the $z_{\mathrm{abs}}=2.3520$ and $z_{\mathrm{abs}}=2.3545$ systems exactly corresponds to that between the subsystems $A$ and $B$.

\subsubsection{System at $\mathrm{Z}_{\mathrm{abs}}=1.7315$}

This system is very similar to that at $z_{\mathrm{abs}}=1.7529$ towards HE1347-2457 (Sect. 3.1.2). A narrow saturated H I $\lambda 1215$ line is blended in the blue and red wings by hydrogen lines from the 

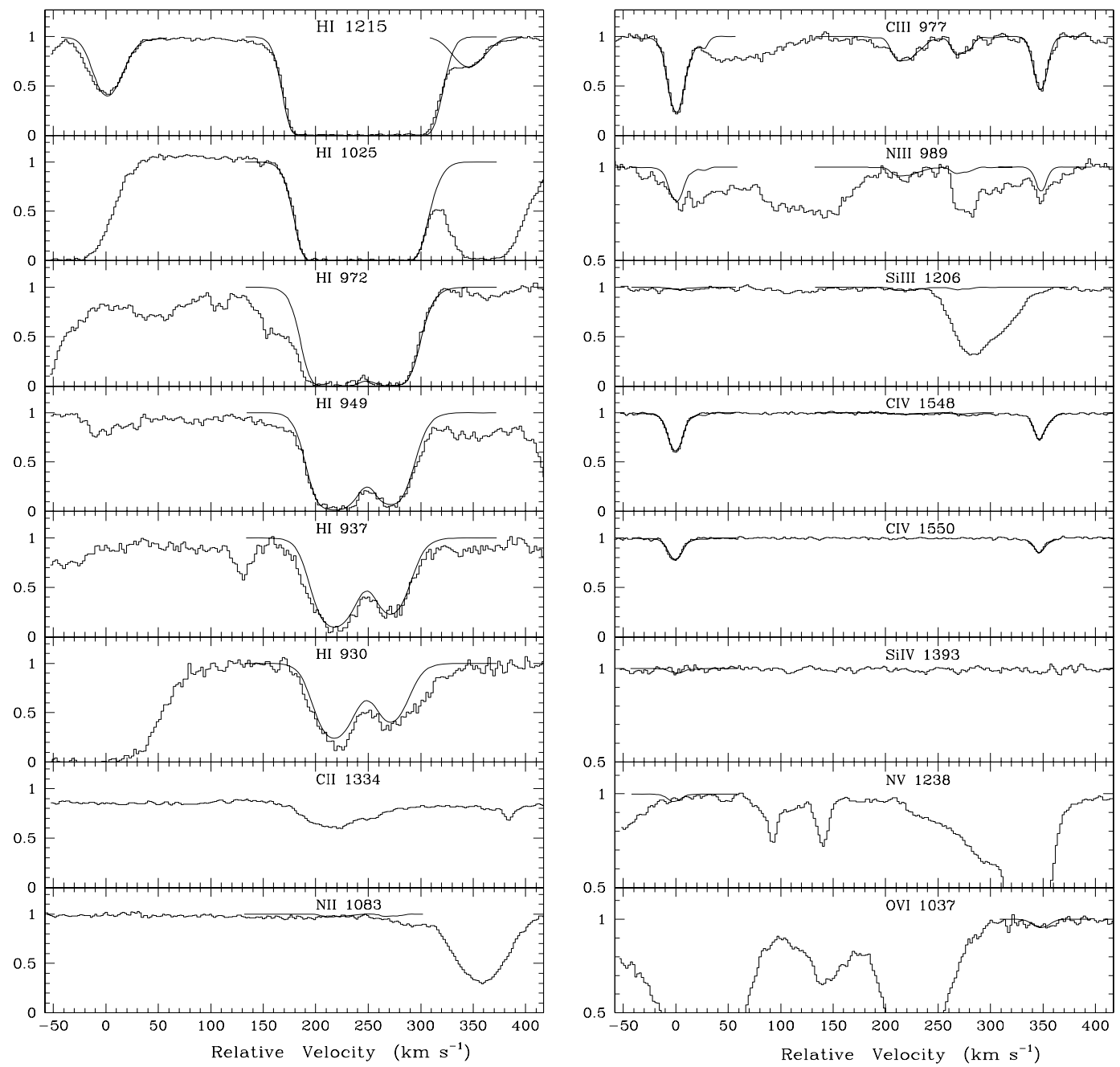

Fig. 8. Same as Fig. 1 but for the complex absorption system at $z_{\text {abs }}=2.4158$ towards HE0151-4326. Three subcomponents cover the ranges: $-30<v<40 \mathrm{~km} \mathrm{~s}^{-1}(A), 140<v<340 \mathrm{~km} \mathrm{~s}^{-1}(B)$, and $300<v<380 \mathrm{~km} \mathrm{~s}^{-1}(C)$.

Ly- $\alpha$ forest (Fig. 10). Both the Si IV lines $1393 \AA$ and $1402 \AA$ coincide with broad intrinsic O VI absorption (HE0151-4326 is a mini-BAL quasar), but the profile of Si IV $\lambda 1393$ can be deconvolved. The line Mg II $\lambda 2796$ is contaminated by telluric absorption. The lines of C IV $\lambda \lambda 1548,1550$ fall in the Ly- $\alpha$ forest, CIV $\lambda 1550$ is blended in the blue wing. Thus it is not clear whether the incomplete coverage is realized or not. In the following all lines are considered as having covering factors of unity. Acceptable shapes of the ionizing spectra are shown in Fig. 11, the derived physical parameters, column densities and the corresponding element abundances are presented in Tables 1-3.

Silicon is considerably depleted relative to carbon, whereas the magnesium to carbon ratio is almost two times higher than the solar value. There is also deficit of iron with respect to carbon. Similar pattern characterized by the strong overabundance of magnesium and deficit of iron (deficit of silicon was not reliably detected), was found in the absorption system at $z_{\text {abs }}=1.7963$ towards HE2347-4342 (\# 10 in Table 1, see also Sect. 3.1.2 in Agafonova et al. 2007). The overabundance of magnesium is observed in AGB-stars and is related to the socalled "hot-bottom" burning (Herwig 2005).

Again, as in the case of the systems at $z_{\text {abs }}=1.7529$ and $z_{\mathrm{abs}}=1.5080$ towards HE1347-2457 (Sects. 3.1.2 and 3.1.3, respectively), the shift of the magnesium lines to $\sim 0.6 \mathrm{~km} \mathrm{~s}^{-1}$ leads to a noticeable improvement of the fitting. The present system shows a narrow but blended line at the position of $\mathrm{NV} \lambda 1238$. Since the expected position of $\mathrm{NV}$ $\lambda 1242$ is blended with a strong forest absorption, the column density obtained from the NV $\lambda 1238$ line is in fact an upper limit. A conservative limit on the nitrogen abundance shows that the overabundance of nitrogen to carbon does not exceed 0.15 dex (1.4 times).

\subsection{Quasar HE0001-2340}

\subsubsection{System at $z_{\text {abs }}=1.6514$}

This system consists of a narrow hydrogen H I $\lambda 1215$ line, saturated and blended in the blue and red wings, and many strong lines of metal ions in different ionization stages (Fig. 12). The simultaneous fitting of all lines assuming constant metallicity throughout the absorber makes it possible to restore the wings of the hydrogen Ly- $\alpha$ line. The line of C II $\lambda 1334$ is blended with a weak forest absorption and can be deconvolved. The expected positions of the $\mathrm{N} v \lambda \lambda 1238,1242$ lines are contaminated with a strong intervening absorption which prevents setting any reasonable limit on the nitrogen abundance. The shift of the 

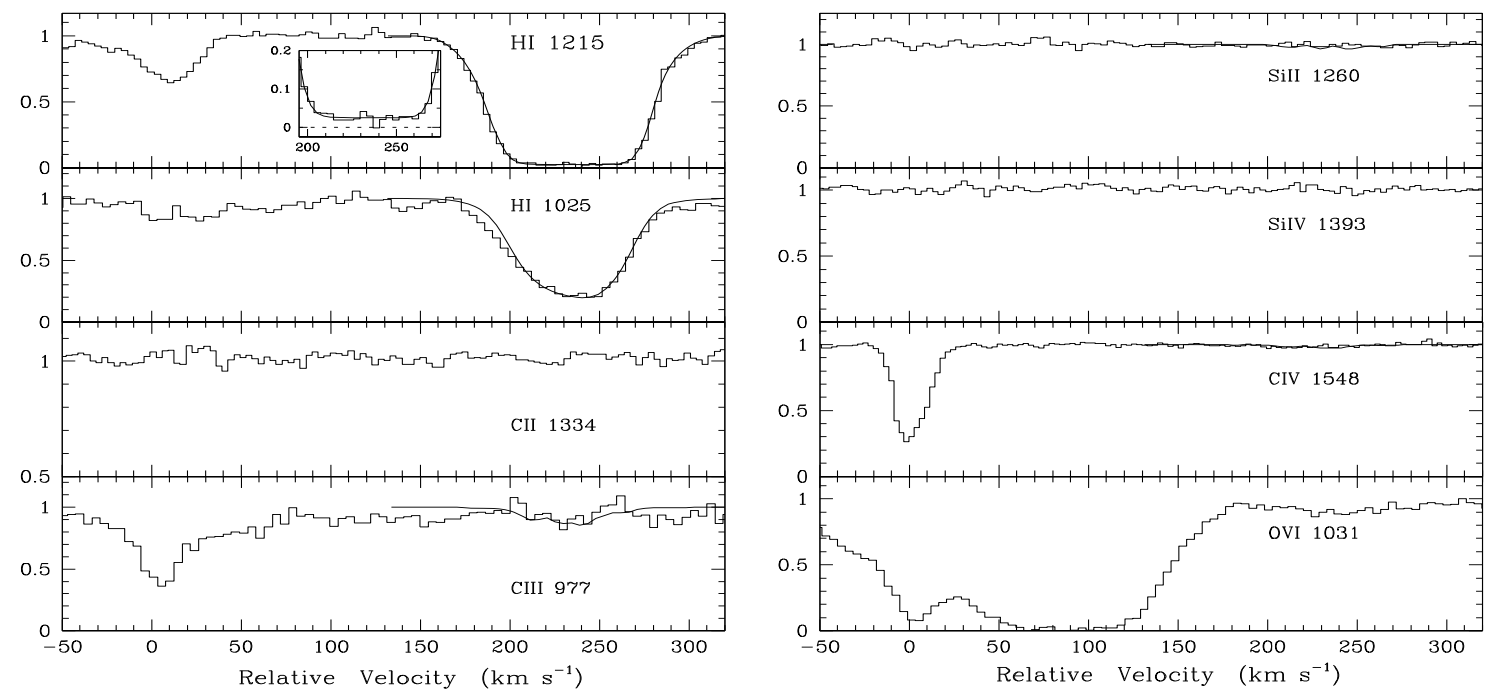

Fig. 9. Same as Fig. 1 but for the complex absorption system at $z_{\text {abs }}=2.3520$ towards Q0329-385. The subsystem at $v=0 \mathrm{~km} \mathrm{~s}^{-1}$ is described in Paper I, Sect. 2.4. The subsystem at $v=230 \mathrm{~km} \mathrm{~s}^{-1}$ has $z_{\mathrm{abs}}=2.3545$.
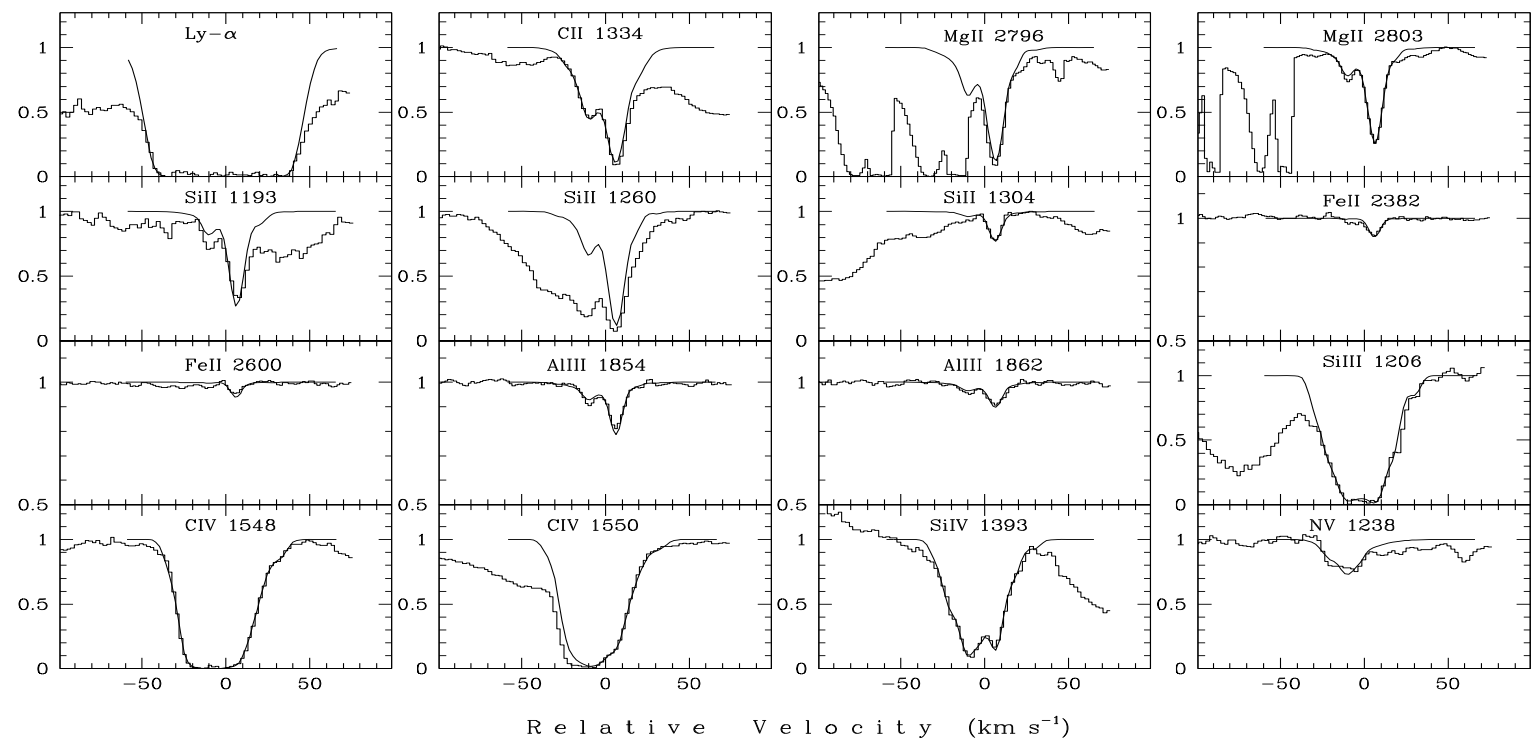

Fig. 10. Same as Fig. 1 but for the $z_{\mathrm{abs}}=1.7315$ system (HE0151-4326).

Mg II $\lambda 2796,2803$ lines by $\sim 0.6 \mathrm{~km} \mathrm{~s}^{-1}$ results in the improved fitting of all low-ionization lines, C II $\lambda 1334$, Si II $\lambda 1526$, and Mg II $\lambda \lambda 2796,2803$ (see Sect. 3.1.2). It cannot be excluded that the magnesium profiles are affected by an incomplete coverage, $C(\mathrm{Mg}$ II $) \sim 0.95-0.98$, but the current quality of the spectral data does not allow us to make unambiguous conclusion. The physical parameters are given in Table 1 . The column densities listed in Table 2 correspond to $C=1$ for all lines.

Very similar ratios $\mathrm{C}$ IV/C II $=$ Si IV $/ \mathrm{Si} \mathrm{II}=\mathrm{Si} \mathrm{III/Si} \mathrm{IV} \sim 1.1$ require a hard ionizing spectrum at $1<E<4$ Ryd with $\alpha \sim 0.8-1$ and with the intensity break at $E=4$ Ryd of about 1 dex (Fig. 13). The resulting element abundances are given in Table 3. Noticeable is an extremely strong depletion of silicon to carbon and magnesium, $[\mathrm{Si} / \mathrm{C}, \mathrm{Mg}] \sim-0.9$. The line Fe II $\lambda 2382$ falls in the spectral region with many weak telluric lines, so it is not clear whether the absorption at the expected position of this lines is indeed due to Fe II (no other iron lines are available). In any case, even an upper limit on the iron content shows that iron is depleted as well, $[\mathrm{Fe} / \mathrm{C}]<-0.3$. In contrast to all systems described above the present one has a deficit of aluminium: $[\mathrm{Al} / \mathrm{C}]<-0.6$. A continuum window at the expected position of the O I $\lambda 1302$ line allows us to set a very conservative limit on the oxygen abundance, $[\mathrm{O} / \mathrm{H}]<0.5$, which excludes any overabundance of oxygen to carbon. The linear size along the line-of-sight is small with an upper limit (the intergalactic $J_{912}$ intensity is assumed) of $L<30$ pc.

\subsubsection{System at $z_{\mathrm{abs}}=1.5770$}

There are three systems - at $z_{\text {abs }}=1.5770,1.5810$, and $1.5855-$ located quite closely in the radial velocity range $(\Delta v \sim$ $1000 \mathrm{~km} \mathrm{~s}^{-1}$ ) and showing a similar set of strong and narrow lines of different metal ions. However, in the two latter systems most lines are blended, so the quantitative analysis is possible only for the former absorber which is shown in Fig. 14.

From silicon lines, only Si III $\lambda 1206$ and Si IV $\lambda 1393$ are present, SiIV $\lambda 1402$ is blended with deep forest absorption. Both lines fall in the Ly- $\alpha$ forest range and strictly speaking should be considered as upper limits (although no metal contaminants were found). A narrow absorption at the position of $\mathrm{N} \mathrm{V}$ $\lambda 1238$ is very probably due to this ion (no metal candidates for 


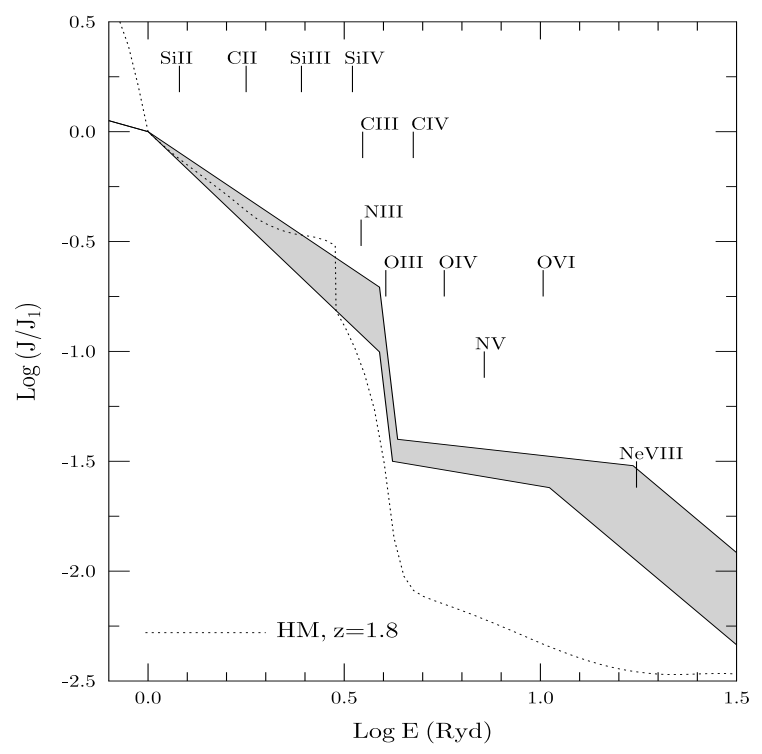

Fig. 11. Same as Fig. 2 but for the $z_{\text {abs }}=1.7315$ system (HE0151-4326).

blending), its red wing is blended with shallow forest line, but can be deconvolved. Mg II $\lambda 2796$ coinsides with a telluric line, weak absorption at the position of Mg II $\lambda 2803$ can also be telluric.

For any type of the tried ionizing spectra the carbon content comes out extremely high - above 10 solar values. The apparent profile of Ly- $\alpha$ is inconsistent with the assumption of constant metallicity throughout the absorber (see synthetic profiles in Fig. 14). Note that similar inconsistency between profiles of hydrogen lines and lines of metals was also detected in Paper I for two absorbers with very high metallicities: the system at $z_{\text {abs }}=2.898$ towards HE2347-4243, and the system at $z_{\text {abs }}=$ 2.352 towards Q0329-385. Both of them are associated.

The physical parameters and column densities are given in Tables 1-3. At such high metallicities as obtained for the $z_{\mathrm{abs}}=$ 1.5770 system the dependence of ion fractions on the absolute metal abundances (which are unknown) overrides the dependence on the SED, so it is not possible to bound the acceptable range of spectral shapes. However, UVB spectra which are softer at $E>4$ Ryd than the model spectrum for $z \sim 2$ of Haardt \& Madau (1996) should be probably excluded since at $U$ corresponding to the observed ratio $\mathrm{C}$ II/C IV they do not reproduce the observed upper limits on Si III and Si IV and give an extremely large (more than one magnitude) overabundance of nitrogen to carbon. The previously described system at $z_{\text {abs }}=$ 1.6514 for which the spectral shape can be restored is detached in the velocity space by $\sim 8000 \mathrm{~km} \mathrm{~s}^{-1}$. In principle, such velocity dispersions could be possible if absorbers were ejected by a quasar located transverse to the line-of-sight. In any case, for all types of trial spectra silicon remains considerably underabundant to carbon, $[\mathrm{Si} / \mathrm{C}]<-0.7$. Also for all spectra, the deconvolved N v $\lambda 1238$ line leads to overabundance of nitrogen to carbon. The value of [N/C] depends on the softness at $E>4$ Ryd: the softer the UVB spectrum the higher [N/C].

Extreme high abundances of carbon and nitrogen might be artificial because of non-equilibrium ionization (H I overionized) in the system considered. However, more plausible explanation seems to be a really $\mathrm{H}$-deficient gas from the inner parts of the envelopes of the AGB-stars. The low ratio of silicon to carbon and nitrogen, $[\mathrm{Si} / \mathrm{C}, \mathrm{N}]<-1$, can be explained by the enhancement of $\mathrm{C}$ and $\mathrm{N}$ due to the dredge-up processes and by the depletion of silicon into dust.

\section{Discussion}

\subsection{Abundance patterns of high metallicity gas}

In the present paper we considered 7 optically-thin systems with solar to oversolar metallicities. Thus, together with previously described absorbers, total sample of metal-rich systems consists of 11 absorbers with redshifts $1.5<z<2.9$ identified in spectra of 6 quasars. To simplify the reading, in the following the systems will be referred to by their numbers as given in Tables 1 and 3. Metallicity is indicated on base of the measured abundance of carbon.

The majority of the systems except \# 9 (Tables 2, 3) show abundance patterns which - in spite of being different - can nevertheless be related to outflows from low and intermediate mass stars $\left(1 M_{\odot}<M<10 M_{\odot}\right)$ in their post-main sequence evolution stages.

The observed diversity of patterns supports the assumption that most metal-rich absorbers have very small sizes $(L \sim$ $10-500 \mathrm{pc})$ as compared with the intergalactic clouds, i.e. absorbers are formed by outflows from only a few stars (or even from a single one).

The system \# 9 shows extreme high metal content exceeding 10 solar values. It is not clear whether the apparent metallicity is artificially boosted due to the overionized state of $\mathrm{H} \mathrm{I}$ or it is intrinsic and thus indicating really hydrogen-deficient gas (see Sect. 2.3.1 in Paper I). In any case, the overabundance of oxygen to carbon points to $\mathrm{SNe}$ II as the main source of gas enrichment. The presence of only one SNe II-enriched system in our sample is probably a selection effect: in quasar spectra there is a nonnegligible population of absorbers revealing strong C IV lines along with weak H I absorption (see, e.g., Schaye et al. 2007). These systems may indeed contain high metallicity gas enriched prevalently by SNe II, but an accurate quantitative analysis is in most cases prevented by the absence of lines in subsequent ionization stages.

Figure 15 shows the ratio $[\mathrm{N} / \mathrm{C}]$ as a function of the carbon abundance $[\mathrm{C} / \mathrm{H}]$ which in the present case serves as an indicator of the overall metallicity. No positive correlation of [N/C] with metallicity is seen: at any value of $[\mathrm{C} / \mathrm{H}]$ nitrogen can be either over- or underabundant. Similar findings were reported for planetary nebulae (Aller \& Czyzak 1983; Perinotto 1991; Richer $\&$ McCall 2008) and for high-metallicity gas near AGN (Fields et al. 2005). This indicates that the nitrogen enrichment occurs quite irregular and is probably governed by many processes. On the other hand, the spread of the nitrogen contents can be considered as an additional argument in favor of a very small linear size of the metal-rich absorbers (formed by outflows from only a few stars).

Absorbers \# 1, 8, 9 and 11 belong to the so-called associated, or proximate systems, i.e. they are located close to the background quasar and are formed in gas ejected from the host galaxy. All other systems are formally intervening. However, they demonstrate same physical properties as the above associated - compare, for example, \# 1 and $2, \# 8$ and 3 , \# 11 and 4. Two conclusions follow. Firstly, many metal-rich intervening systems originate in gas expelled from the quasar host galaxies located transverse to the line of sight. This is also supported by the direct observations of HE2347-4342 where transverse quasars were detected just at the redshifts of such systems (Worseck et al. 2007). Secondly, associated systems not nessesary originate in gas ejected from the circumnuclear regions - they can be interstellar objects (fragments of star/PN envelopes, diffuse clouds etc.) entrained by the quasar wind and transported into the intergalactic space. However, metal 


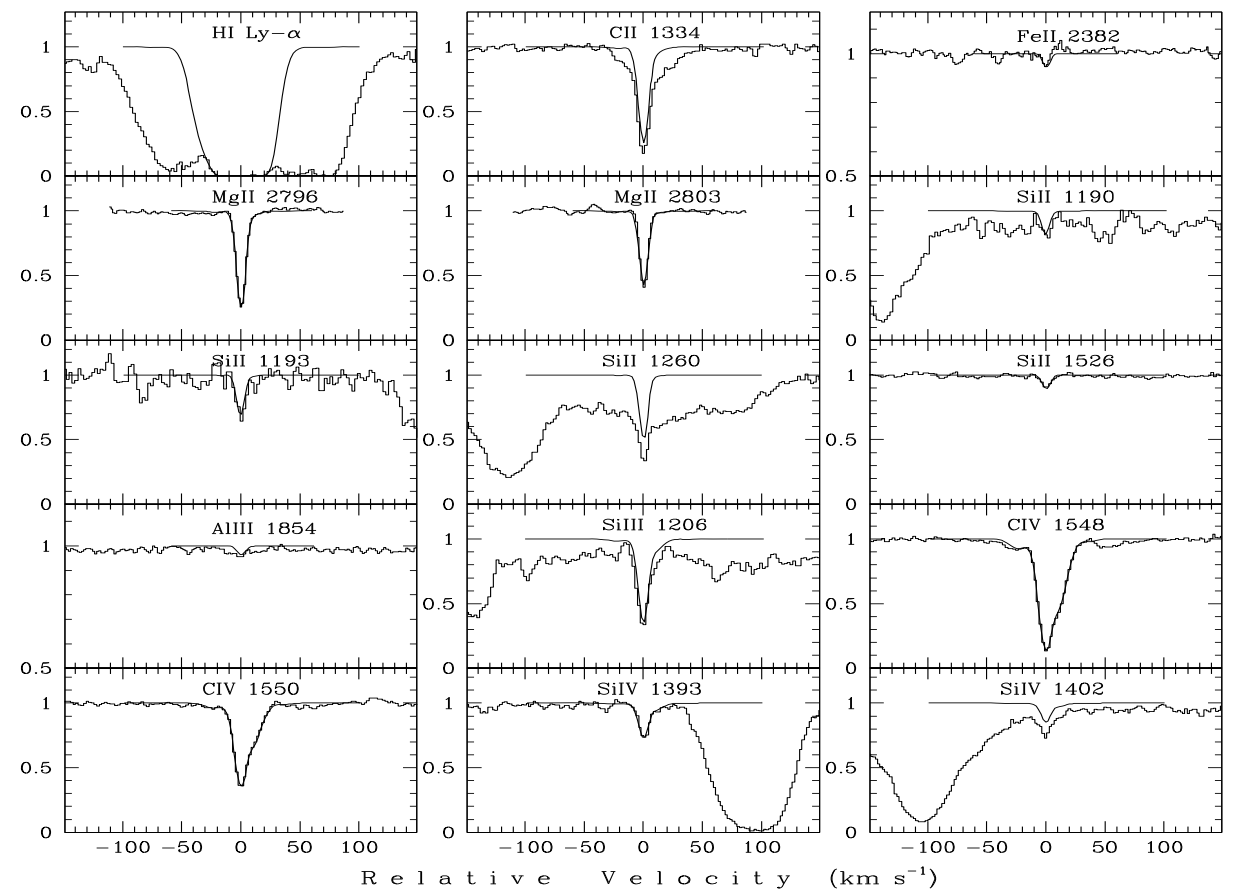

Fig. 12. Same as Fig. 1 but for the $z_{\text {abs }}=1.65136$ system (HE0001-2340).

abundances obtained from the associated systems are often used to verify models of quasar chemical evolution (e.g. D'Odorico et al. 2004; Fechner \& Richter 2009). Obviously, before making this, the nature of a particular associated system should be clarified.

\subsection{Depletion of silicon into dust}

In Sect. 3.1.1 we argued that the deficit of silicon to carbon obtained for the metal-rich gas may be due to condensation of silicon into dust in the envelopes of post-main sequence stars and the subsequent gas-dust separation in the stellar/galactic wind. Here we consider some aspects of silicon depletion into dust in more detail.

In the oxygen-rich environment, $\mathrm{C} / \mathrm{O}<1$, the main dust species are iron and silicates consisting of olivine $\mathrm{Mg}_{2 x} \mathrm{Fe}_{2(1-x)} \mathrm{SiO}_{4}$, pyroxen $\mathrm{Mg}_{x} \mathrm{Fe}_{1-x} \mathrm{SiO}_{3}$ and quartz $\mathrm{SiO}_{2}$ (Gail \& Sedlmayr 1999; Jeong et al. 2003; Ferrarotti \& Gail 2006), with olivine and pyroxen observed mostly in iron-free (i.e. $x=1$ ) forms (Jaeger et al. 1998; Waters et al. 1998; Draine 2003; Gutenkunst et al. 2008; Perea-Calderón et al. 2009). Although processes of dust growth are far from being clear in full detail, all reactions related to the silicate dust formation require the presence of the molecule $\mathrm{SiO}$. This molecule is indeed observed in the envelopes of oxygen-rich AGB- and RSG-stars (van Loon et al. 2008; Sloan et al. 2008). Contrary to the previous standpoint that $\mathrm{SiO}$ polimerizes at low temperature $\sim 600 \mathrm{~K}$ and therefore cannot form so-called seed nuclei (Jeong et al. 2003), recent results have shown that $\mathrm{SiO}$ nucleates in fact at significantly higher temperature $T \sim 1200 \mathrm{~K}$ and forms small $\mathrm{SiO}$ grains over which the other dust components start to condense at continuously lower $T$ (Nuth \& Fergusson 2006). Thus, at the initial stages of dust formation the most depleted elements are expected to be oxygen and silicon, with magnesium and iron following at the later stages. Observational evidences of strong magnesium gradients throughout some planetary nebulae along with more or less uniform depletion of silicon can be

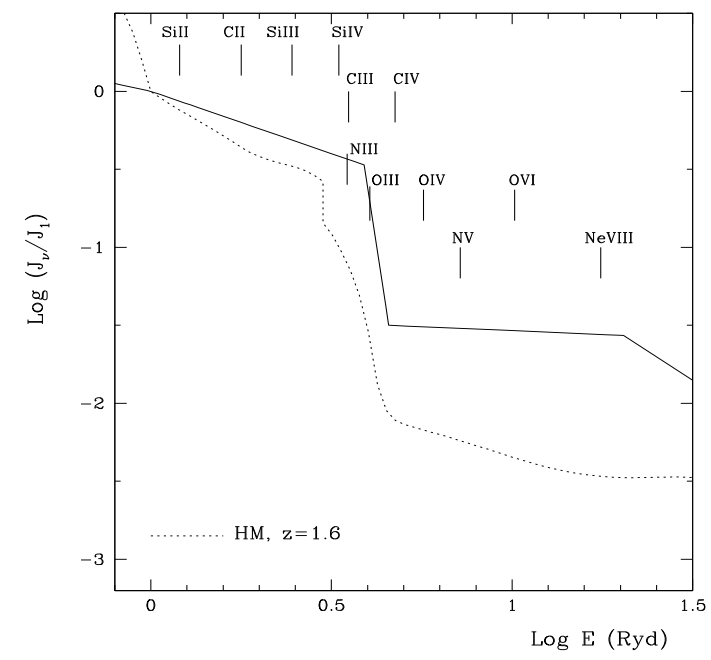

Fig. 13. The ionizing spectrum (solid line) corresponding to the ionization state observed in the $z_{\mathrm{abs}}=1.65136$ system (HE0001-2340). The intergalactic ionizing spectrum at $z=1.6$ modeled by Haardt \& Madau (1996) is shown by the dotted line. The spectra are normalized as in Fig. 2.

considered as a support for this picture (Péquignot \& Stasińska 1980; Harrington \& Marionni 1981; Middlemass 1988).

We note that systems with $[\mathrm{Si} / \mathrm{C}]<0$ (\# 2, 5, 6 in Table 3$)$ do not show deficit of silicon to iron which is not in line with usual assumption that iron should be stronger depleted into dust than silicon. In principle, it is possible that the depletion of iron is stronger in our systems as well: the intrinsic iron content is unknown and it can be enhanced relative to other elements as was obtained, e.g., for the absorbers \# 3 and 8 (and is observed in giant stars in local dwarf galaxies - see Sect. 3.1.3). However, the growth of dust grains includes many stages and is strongly affected by the environmental conditions such as gas 


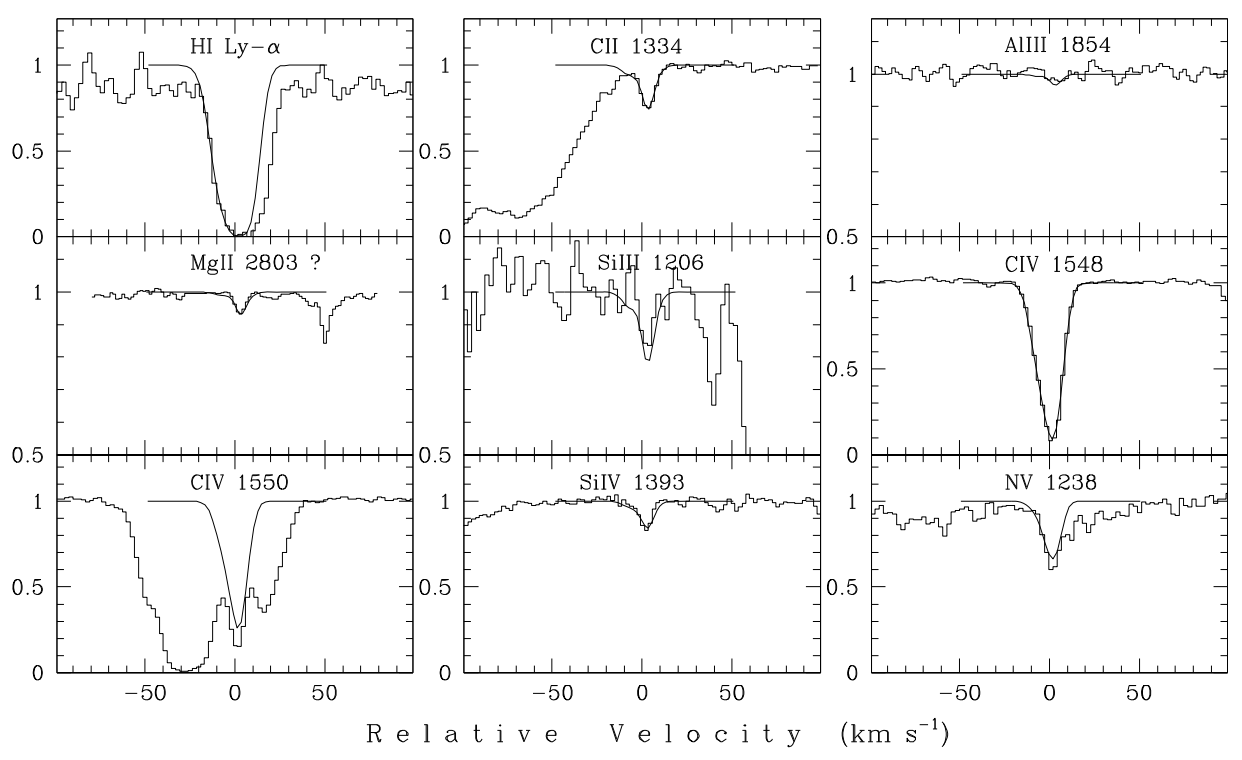

Fig. 14. Same as Fig. 1 but for the $z_{\text {abs }}=1.5770$ system (HE0001-2340). Identification of the $\mathrm{Mg}$ II $\lambda 2803$ line is putative since the stronger component $\mathrm{Mg}$ II $\lambda 2796$ is blended with telluric absorption.

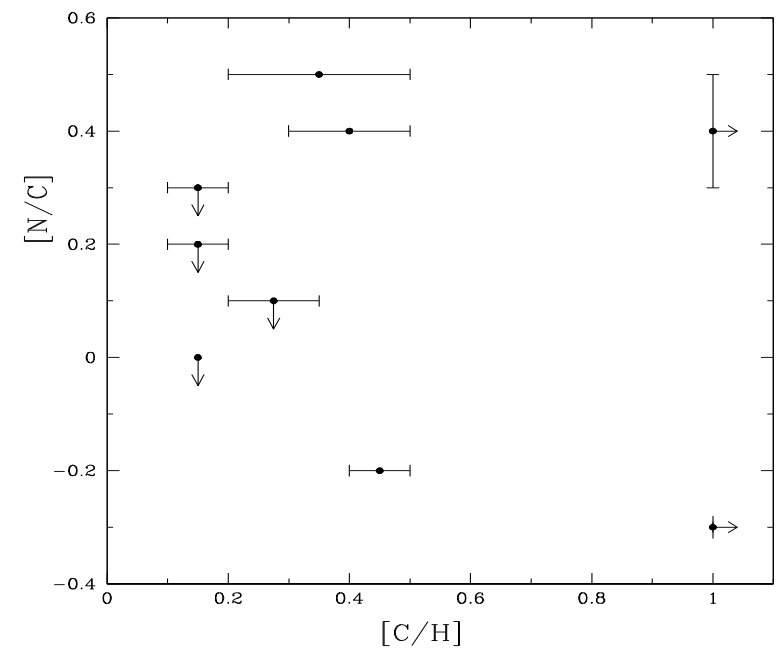

Fig. 15. Relative ratio $[\mathrm{N} / \mathrm{C}]$ versus metallicity index $[\mathrm{C} / \mathrm{H}]$ for metalrich absorbers (see Table 3).

metallicity, temperature, star pulsations etc. Under some conditions iron becomes more depleted than other elements, under other - does not. Model calculations of the dust growth processes in AGB-stars by Ferrarotti \& Gail (2006) show that the relative depletion of $\mathrm{Si}$ and $\mathrm{Fe}$ depends on the metallicity and initial stellar mass, with silicate dust prevalent over iron dust in high-metallicity (solar to oversolar) AGB-stars with masses $M<2 M_{\odot}$ or in massive AGB-stars $M \gtrsim 4 M_{\odot}$ experiencing hotbottom burning. The hot-bottom burning is considered also as the cause of the enhanced content of heavy magnesium isotopes ${ }^{25} \mathrm{Mg}$ and ${ }^{26} \mathrm{Mg}$ (Herwig 2005) indications of which were detected in the absorbers \# 2, 3, 5, and 6 (Sects. 3.1.2, 3.1.3, 3.2.2, 3.3.1, and 3.3.2, respectively). Thus, we may conclude that the absorbers in question are formed mainly from gas expelled from massive AGB-stars.

Note in passing that among metal-rich DLAs and LLSs, there are objects with both low $[\mathrm{Si} / \mathrm{Fe}]:[\mathrm{Zn}]=0.28,[\mathrm{Si} / \mathrm{Fe}]<-0.5$ (Péroux et al. 2008), $[\mathrm{Zn}]=0.25,[\mathrm{Si} / \mathrm{Fe}]<-0.4$ (Meiring et al. 2007), and high $[\mathrm{Si} / \mathrm{Fe}]:[\mathrm{Zn}]>0.86,[\mathrm{Si} / \mathrm{Fe}]=0.75$

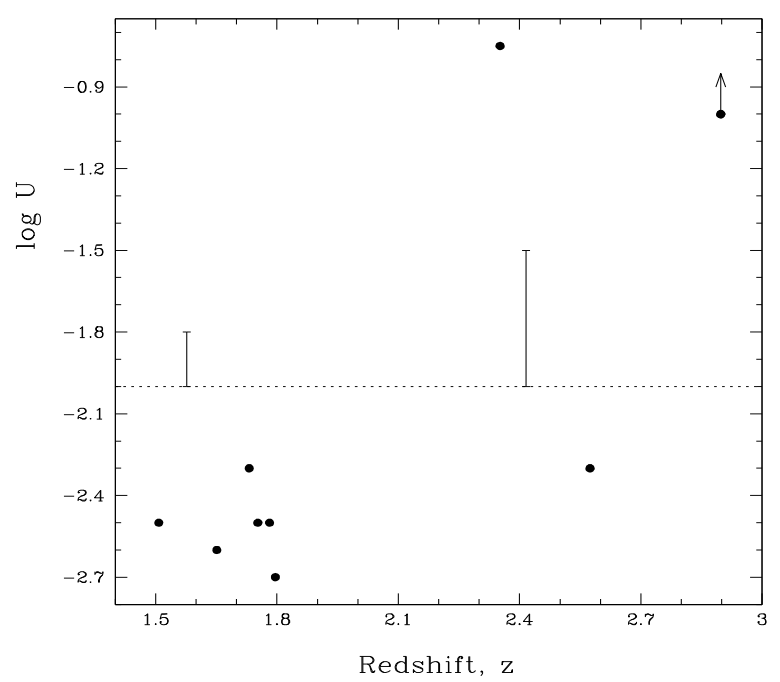

Fig. 16. Ionization parameter $\log U$ estimated from metal-rich absorbers with redshifts $1.5<z<2.9$ (see Table 2). The dashed line separates absorbers with low and high $U$-values.

(Meiring et al. 2008), $[\mathrm{Zn}]=0.12,[\mathrm{Si} / \mathrm{Fe}]=0.7$ (Prochaska et al. 2006). Additionally, many DLAs shows differential depletion into dust with values $[\mathrm{Si} / \mathrm{Fe}]$ varying an order of magnitude from one component to another (Centurión et al. 2003; Péroux et al. 2006; Quast et al. 2008).

\subsection{Evolutional effects in distribution of metal-rich absorbers}

On base of the observed lines, the metal-rich absorbers can be divided into two distinct groups: systems revealing strong lines of both low (C II, Mg II, Si II) and high (C III, N III, Al III, Si III, C IV, Si IV) ionization species (\# 1, 2, 3, 5, 6, 8, 10) and systems without or with weak low-ionization lines $(\# 4,7,9,11)$. The first group has the mean ionization parameter $\log U<-2$, whereas the second one is characterized by $\log U>-1.5$. In Fig. 16, the $U$-values are plotted against the redshifts of the systems for 
which they are obtained. The absence of systems from the second group at $z<2.3$ in our sample is explained by pure selection effects: the important for these systems lines of C III $\lambda 977$, N III $\lambda 989$, and O VI $\lambda \lambda 1032,1037$ are shifted at $z<2.3$ to the unobservable or very noisy wavelength range, and the remaining H I $\lambda 1215$, C IV $\lambda \lambda 1548,1550$, and eventually N V $\lambda \lambda 1238,1242$, and Si IV $\lambda \lambda 1393,1402$ do not allow to fix the ionization parameter and, hence, to derive accurate element contents.

As for low-ionization systems, we find a single system at $z>2$ (\# 1 with $z_{\text {abs }}=2.5745$ ) and six systems at $z<2$. In contrast to the high-ionization group, this result is not affected either by selection criteria for absorbers or by the wavelength coverage of available quasar spectra (low redshift boundary $z_{\mathrm{abs}}=1.5$ is determined by the optical limit to observe H I 21215 ) and, thus, reflects a real distribution of low- $U$ absorbers over redshift.

The epoch $z \sim 2$ is known to represent a peak activity in both the cosmic star formation rate and the assembly of galaxies and it is also characterized by the maximum space density of the luminous quasars (Fan et al. 2001; Dickinson et al. 2003; Chapman et al. 2005; Rudnick et al. 2006). Galaxies at $z \sim 2$ reveal star formation rates of $10-300 M_{\odot} \mathrm{yr}^{-1}$ and stellar masses $M_{*} \sim 10^{9}-10^{11.5} M_{\odot}$ (Genzel et al. 2008, and references cited therein). The estimates of the comoving number density of the star-forming galaxies from different surveys (Chen et al. 2003; Adelberger et al. 2005; Sumiyoshi et al. 2009; Cristóbal-Hornillos et al. 2009) provide a density $n_{g}$ of a few $10^{-3} \mathrm{~h}^{3} \mathrm{Mpc}^{-3}$.

Taking into account the detected jump in the number of lowionization absorbers at $z_{\mathrm{abs}}<2$, it is interesting to compare the space density of these absorbers to the space density of galactic population at $z \sim 2$.

At $z=2$, the H I Ly- $\alpha$ is shifted to $3647 \AA$. Among the studied spectra of 20 QSOs, only half of them cover the wavelength range $\lambda<3647 \AA$, thus allowing us to identify low-ionization absorbers at $z<2$. In spectra with the coverage $\lambda<3647 \AA$, the probability to find at least one low-ionization metal-rich absorber clearly exceeds $50 \%$. Assuming that the mean linear size of the absorber is $\sim 50 \mathrm{pc}$, the chance that a random sightline passes through a metal-rich cloud requires the comoving number density of the absorbers $n_{\mathrm{a}} \sim 1 / L^{2} D$. With a typical distance lag $D \sim 10 \mathrm{Gpc}(z \sim 1.8)$, the observed absorber frequency is realized if the comoving density of these systems is $n_{\mathrm{a}} \sim 4 \times 10^{4} \mathrm{Mpc}^{-3}$. Here the comoving density of star-forming galaxies is taken as $n_{g} \approx 2.4 \times 10^{-3} \mathrm{Mpc}^{-3}(h=0.7)$ which is in line with Chen et al. (2003). Then there are $2 \times 10^{7}$ metal-rich absorbers in the vicinity of each star-forming galaxy at $z \lesssim 2$. This estimate is quite rough because of the unknown geometric parameters of the clouds and the uncertainties in the galactic counting statistics. However, it corresponds by an order of magnitude to recent results on the Milky Way halo where a population of more than $10^{8}$ weak metal-line absorbers located in circumgalactic environment was detected (Richter et al. 2009). Richter et al. select their systems on base of the presence of a weak O I $\lambda 1302$ line which supposes the ionization parameter $U$ of about $\log U \sim-3$ (cf, our system \# 10). The physical parameters of these Milky Way 'gas wisps' are very much alike to their high-redshift counterparts: the hydrogen column density $N(\mathrm{HI})$ ranges from $2 \times 10^{16} \mathrm{~cm}^{-2}$ to $3 \times 10^{18} \mathrm{~cm}^{-2}$, the hydrogen volume density $n_{\mathrm{H}} \sim 0.02-0.3 \mathrm{~cm}^{-3}$ (if clouds are located at the distance $50 \mathrm{kpc}$ from the Galactic center), the absorber thicknesses are $L \approx 0.2-130 \mathrm{pc}$, and the metallicities are $\sim 0.1-1$ solar.
Finally, to estimate the mass of the clouds, we assume that they are homogeneous spheres of radius $R$. Then,

$$
M_{\mathrm{a}}=\frac{4}{3} \pi R^{3} n_{\mathrm{H}} \mu \mathrm{m}_{\mathrm{H}} \approx 0.1 \mu\left(\frac{n_{\mathrm{H}}}{\mathrm{cm}^{-3}}\right)\left(\frac{R}{\mathrm{pc}}\right)^{3} M_{\odot},
$$

where $\mu$ is the mean molecular weight of the gas, and $m_{\mathrm{H}}$ is the mass of the hydrogen atom.

For ionized gas of solar metallicity $(X: Y: Z=$ 0.710:0.265:0.025 by mass), $\mu=0.62$. With $n_{\mathrm{H}} \sim 10^{-2} \mathrm{~cm}^{-3}$ and $R \sim 25 \mathrm{pc}$, we obtain for a single cloud a typical mass of $M_{\mathrm{a}} \sim 10 M_{\odot}$, i.e. low-ionization metal-rich absorbers can indeed be produced by only a few intermediate-mass stars. For a star-forming galaxy with $M_{*} \sim 2 \times 10^{10} M_{\odot}$, the total mass $M_{\text {tot }}$ of all metal-rich absorbers reaches $M_{\mathrm{tot}} \sim 0.01 M_{*}$.

The peak star forming activity at $z \sim 2$ is usually explained by the presence of large amounts of gas supplied either by mergers or by smooth gas accretion onto galaxies along the largescale filaments, with probable prevalence of the second mechanism (Genel et al. 2008; Shapiro et al. 2008; Genzel et al. 2008). Our data show that galaxies at $z \lesssim 1.8$ have already undergone stellar evolution sufficient to provide high (solar to oversolar) level of metallicity for the large population of stars. The buildup of metals requires many generations of stars. Estimations from SINFONI survey of galaxies at $z \sim 2$ show that starforming discs experienced constant star formation rates over at least 0.5 Gyr (Daddi et al. 2007). This means more than ten generations for intermediate mass stars - probably enough time to acquire high metallicity.

The high metal content has positive effect on the star massloss rates through driving the outflow both by radiative acceleration in resonance lines of metal ions and by radiation pressure on the dust, the formation of which is in turn boosted in the metalrich environment. Thus, at high metallicity the recycling of matter occurs more rapid leading to constantly large amount of gas present in the interstellar space. On the other hand, starbursts and possible AGNs in the central regions favor the formation of the large-scale winds which expel gas from galaxies. At some redshift, more gas is expelled as returned resulting in decline of both star forming and AGN activities. This point occurs somewhere below redshift 1.5. Unfortunately, wavelength coverage of optical telescopes limits redshifts of absorption systems suitable for quantitative analysis - below $z=1.5$ the hydrogen $\mathrm{H}$ I $\lambda 1215$ line is not seen and, hence, accurate metallicity of the absorbing gas cannot be determined. Commissioning of the Cosmic Origins Spectrograph - the high-resolution UV spectrograph with wavelength coverage from 1100 to $3200 \AA$ - and the World Space Observatory (WSO)-UV will change the situation allowing to probe high-metallicity systems at redshifts below 1.5 .

\section{Summary and conclusions}

We report on the analysis of 11 metal-rich absorption systems with redshifts $1.5<z<2.9$ identified in spectra of 6 distant quasars. Due to a special selection of the absorption systems and the advanced methods to solve the inverse spectroscopy problem the obtained abundance patterns can be considered as reliable. On base of these patterns, we identify types of stars responsible for the enrichment of the absorbing gas. The main results are as follows:

1. The majority of the described metal-rich absorbers ( 10 from 11) reveal abundance patterns which - in spite of being different - can be attributed to the outflows from low and intermediate mass stars (LIMS) in their post-main sequence 
evolutional stages (red giant branch and asymptotic giant branch). One absorber is enriched prevalently by SNe II. A small number of SNe II-enriched objects does not represent real statistics, but is a consequence of selection criteria. Diversity of observed patterns points to small linear sizes of the metal-rich absorbers, i.e. every cloud can be formed by outflows from only a few (or even from a single) star(s).

2. Several absorbers show a significant deficit of silicon with respect to other elements. This deficit can be explained by the depletion of $\mathrm{Si}$ into dust in the oxygen-rich envelopes of the post-main sequence stars and the subsequent dust-gas separation in the galactic/quasar wind.

3. If lines of $\mathrm{Mg}$ II $\lambda \lambda 2796,2803$ are centered using the laboratory wavelengths which correspond to the solar isotopic ratio ${ }^{24} \mathrm{Mg}:{ }^{25} \mathrm{Mg}:{ }^{26} \mathrm{Mg}=79: 10: 11$, then profiles of all lowionization lines ( $\mathrm{C}$ II, Mg II, Si II, Fe II) cannot be fitted selfconsistently with a required $\chi_{\min }^{2} \sim 1$. The quality of fitting improves significantly if centering of the Mg II lines occurs using slightly shorter wavelengths which correspond to an enhanced content of heavy isotopes ${ }^{25} \mathrm{Mg}$ and ${ }^{26} \mathrm{Mg}$ in the absorbing gas. Although the quality of the available quasar spectra does not allow to estimate accurate isotopic composition of magnesium, the enhancement of heavy $\mathrm{Mg}$ isotopes in the systems at $z_{\mathrm{abs}}=1.7529,1.7315,1.6514$, and 1.5080 seems to be very probable. Such an enhancement is predicted for AGB-stars with masses $M \gtrsim 4 M_{\odot}$ which experience a so-called hot-bottom burning.

4. The abundance of nitrogen does not show correlation with metallicity: at any value of $[\mathrm{C} / \mathrm{H}]$ nitrogen can be both overand underabundant to carbon. This means that the nitrogen enrichment occurs quite irregularly and is governed by several processes.

5. Among metal-rich systems, a group characterized by low ionization parameter, $\log U<-2.3$, can be singled out. At $z>2$, we find only one low- $U$ system $\left(z_{\text {abs }}=2.5745\right)$, whereas at $z<2$ six such systems are detected. This result is not affected by any selection criteria and reflects the real redhsift distribution of the low-ionization metal rich systems. The comparison of the number densities of metal-rich absorbers and star-forming galaxies at $z \sim 2$ shows that there should be $\sim 10^{7}-10^{8}$ such absorbers around each galaxy. This coincides with the number of small absorbing clouds detected recently around the Milky Way. The redshift $z \sim 2$ is characterized by peak values of the star-formation rates and the quasar luminosity function which are currently explained by the presence of large amounts of gas in the interstellar space of high-redshift galaxies due to mergers and rapid gas accretion along the large-scale filaments, with the second option more preferable by recent observations of the star-forming galaxies at $z \sim 2$. A high metallicity can be another cause for the peak activities at this redshift: large amount of metals in the envelopes of LIMS boosts the massloss from stars both due to radiative acceleration in resonance lines of metal ions and by radiation pressure on the dust grains. This leads to a rapid recycling of matter and, hence, to a constantly high amount of gas present in the interstellar space. When the amount of gas expelled from a galaxy due to the large-scale wind exceeds the amount of gas supplied by accretion and stellar winds, the star-forming and AGN activities decline. In order to study these processes through the analysis of metal-rich absorbers - which turned out to be quite reliable indicators of cosmic evolution - joint optical/UV observations of quasar spectra are needed. This is expected to occur with future Cosmic Origine Spectrograph and WSO-UV.

Acknowledgements. S.A.L. and I.I.A. gratefully acknowledge the hospitality of Osservatorio Astronomico di Trieste, Hamburger Sternwarte, and the Shanghai Astronomical Observatory while visiting there. This research has been supported by the RFBR grant No. 09-02-00352, and by the Federal Agency for Science and Innovations grant NSh 2600.2008.2. J.L.H. is supported by the National Science Foundation of China No. 10573028, the Key Project No. 10833005, the Group Innovation Project No. 10821302, and by the 973 program with No. 2007CB815402.

\section{References}

Adelberger, K. L., Steidel, C. C., Pettini, M., et al. 2005, ApJ, 619, 697 Agafonova, I. I., Centurión, M., Levshakov, S. A., et al. 2005, A\&A, 441, 9 Agafonova, I. I., Levshakov, S. A., Reimers, D., et al. 2007, A\&A, 461, 893 Aller, L. H., \& Czyzak, S. J. 1983, ApJS, 51, 211

Aracil, B., Petitjean, P., Pichon, C., et al. 2004, A\&A, 419, 811

Asplund, M., Grevesse, N., \& Sauval, A. J. 2004, Nucl. Phys. A, 777, 1

Bashkin, S., \& Stoner, J. O., Jr. 1975, Atomic Energy Levels and Grotrian Diagrams (Amsterdam: North-Holland Pub. Company)

Bonifacio, P., Sbordone, L., Marconi, G., et al. 2004, A\&A, 414, 503

Calura, F., \& Matteucci, F. 2004, MNRAS, 350, 351

Centurión, M., Molaro, P., Vladilo, G., et al. 2003, A\&A, 403, 55

Chapman, S. C., Blain, A. W., Smail, I., et al. 2005, ApJ, 622, 722

Chen, H.-W., Marzke, R. O., McCarthy, P. J., et al. 2003, ApJ, 586, 745

Cohen, J. G., Huang, W., Udalski, A., et al. 2008, ApJ, 682, 1029

Cristóbal-Hornillos, D., Aguerri, J. A. L., Moles, M., et al. 2009, ApJ, 696, 1554

Curdt, W., Brekke, P., Feldman, U., et al. 2001, A\&A, 375, 591

Daddi, E., Dickinson, M., Morrison, G., et al. 2007, ApJ, 670, 156

Davies, B., Origlia, L., Kudritzki, R.-P., et al. 2009, ApJ, 696, 2014

Dickinson, M., Papovich, C., Ferguson, H. C., et al. 2003, ApJ, 587, 25

D’Odorico, V., Cristiani, S., Donatella, R., Granato, G. L., \& Danese, L. 2004, MNRAS, 351, 976

Draine, B. T. 2003, ARA\&A, 41, 241

Fabbian, D., Recio-Blanco, A., Gratton, R. G., et al. 2005, A\&A, 434, 235

Fan, X., Strauss, M. A., Schneider, D. P., et al. 2001, AJ, 121, 54

Fardal, M. A., Giroux, M. L., \& Shull, M. 1998, AJ, 115, 2206

Fechner, C., \& Richter, P. 2009, A\&A, 496, 31

Ferland, G. J., Korista, K. T., Verner, D. A., et al. 1998, PASP, 110, 761

Ferrarotti, A. S., \& Gail, H.-P. 2006, A\&A, 447, 553

Fields, D., Mathur, S., Pogge, R. W., et al. 2005, ApJ, 634, 928

Gail, H.-P., \& Sedlmayr, E. 1999, A\&A, 347, 594

Genel, S., Genzel, R., Bouché, N., et al. 2008, ApJ, 688, 789

Genzel, R., Burkert, A., Bouché, N., et al. 2008, ApJ, 687, 59

Gutenkunst, S., Bernard-Salas, J., Pottasch, S. R., Sloan, G. C., \& Houck, J. R. 2008, ApJ, 680, 1206

Haardt, F., \& Madau, P. 1996, ApJ, 461, 20

Hamann, F., \& Ferland, G. 1999, ARA\&A, 37, 487

Harrington, J. P., \& Marionni, P. A. 1981, in The Universe at Ultraviolet Wavelengths: The First Two Yrs. of Intern. Ultraviolet Explorer (Maryland Univ., College Park), 623

Herwig, F. 2005, ARA\&A, 43, 435

Holweger, H. 2001, in Solar and Galactic Composition, ed. R. F. WimmerSchweingruber, AIP Conf. Proc., 598, 23

Hou, J. L., Boissier, S., \& Prantzos, N. 2001, A\&A, 370, 23

Jaeger, C., Molster, F. J., Dorschner, J., et al. 1998, A\&A, 339, 904

Jenkins, E. B., Bowen, D. V., Tripp, T. M., et al. 2005, ApJ, 623, 767

Jeong, K. S., Winters, J. M., Le Bertre, T., et al. 2003, A\&A, 407, 191

Karakas, A. I., \& Lattanzio, J. C. 2003, PASA, 20, 279

Kelly, R. L. 1987, J. Phys. Chem. Ref. Data, 16, 1

Levshakov, S. A., Agafonova, I. I., \& Kegel, W. H. 2000, A\&A, 360, 833 [LAK]

Levshakov, S. A., Agafonova, I. I., Centurión, M., et al. 2003, A\&A, 397, 851

Levshakov, S. A., Molaro, P., D’Odorico, S., et al. 2007, A\&A, 446, 1077

Levshakov, S. A., Agafonova, I. I., Reimers, D., Hou, J. L., \& Molaro, P. 2008, A\&A, 483, 19, Paper I

Liu, Y., Liu, X.-W., Barlow, M. J., et al. 2004, MNRAS, 353, 1251

Marengo, M. 2009, PASA, 26, 365

Meiring, J. D., Lauroesch, J. T., Kulkarni, V. P., et al. 2007, MNRAS, 376, 557

Meiring, J. D., Kulkarni, V. P., Lauroesch, J. T., et al. 2008, MNRAS, 384, 1015

Middlemass, D. 1988, MNRAS, 231, 1025

Middlemass, D. 1990, MNRAS, 244, 294

Molaro, P., Levshakov, S. A., Monai, S., et al. 2008, A\&A, 481, 559

Morton, D. C. 1991, ApJS, 77, 119

Morton, D. C. 2003, ApJS, 149, 205 
Nuth, J. A., III, \& Ferguson, F. T. 2006, ApJ, 649, 1178

Perea-Calderón, J. V., García-Hernández, D. A., García-Lario, P., Szczerba, R., \& Bobrowsky, M. 2009, A\&A, 495, L5

Perinotto, M. 1991, ApJS, 76, 687

Péroux, C., Meiring, J. D., Kulkarni, V. P., et al. 2006, MNRAS, 372, 369

Péroux, C., Meiring, J. D., Kulkarni, V. P., et al. 2008, MNRAS, 386, 2209

Péquignot, D., \& Stasińska, G. 1980, A\&A, 81, 121

Polletta, M., Weedman, D., Hönig, S., et al. 2008, ApJ, 675, 960

Prochaska, J. X., O’Meara, J. M., Herbert-Fort, S., et al. 2006, ApJ, 648, L97

Quast, R., Reimers, D., \& Baade, R. 2008, A\&A, 477, 443

Reimers, D., Köhler, T., \& Wisotzki, L. 1996, A\&AS, 115, 235

Reimers, D., Janknecht, E., Fechner, C., et al. 2005, A\&A, 435, 17

Reimers, D., Agafonova, I. I., Levshakov, S. A., et al. 2006, A\&A, 449, 9

Rich, R. M., Origlia, L., \& Valenti, E. 2007, ApJ, 665, 119

Richer, M. G., \& McCall, M. L. 2008, ApJ, 684, 1190

Richter, P., Charlton, J. C., Fangano, A. P. M., Ben Bekhti, N., \& Masiero, J. R. 2009, ApJ, 695, 1631

Rudnick, G., Labbé, I., Förster Schreiber, N. M., et al. 2006, ApJ, 650, 624

Sandlin, G. D., Bartoe, J.-D. F., Brueckner, G. E., Tousey, R., \& Van Hoosier, M. E. 1986, ApJS, 61, 801

Schaye, J., Carswell, R. F., \& Kim, T.-S. 2007, MNRAS, 379, 1169

Shapiro, K. L., Genzel, R., Förster Schreiber, N. M., et al. 2008, ApJ, 682, 231

Silverman, J. D., Lamareille, F., Maier, C., et al. 2009, ApJ, 696, 396
Simcoe, R. A., Sargent, W. L., \& Rauch, M. 2004, ApJ, 606, 92

Simcoe, R. A., Sargent, W. L., Rauch, M., et al. 2006, ApJ, 637, 648

Sloan, G. C., Kraemer, K. E., Wood, P. R., et al. 2008, ApJ, 686, 1056

Songalila, A., \& Cowie, L. L. 1996, AJ, 112, 335

Stanghellini, L. 2007, in Why Galaxies Care About AGB Stars: Their Importance as Actors and Probes, ed. F. Kerschbaum, C. Charbonnel, \& R. F. Wing (San Francisco: ASP), 456

Sumiyoshi, M., Totani, T., Oshige, S., et al. 2009 [arXiv: 0902 .2064]

Tautvaišienè, G., Geisler, D., Wallerstein, G., et al. 2007, AJ, 134, 2318

van Loon, J. Th., Cohen, M., Oliveira, J. M., et al. 2008, A\&A, 487, 1055

Venn, K. A., Irwin, M., Shetrone, M. D., et al. 2004, AJ, 128, 1177

Vladilo, G. 2002, A\&A, 391, 407

Vladilo, G., \& Péroux, C. 2005, A\&A, 444, 461

Waters, L. B. F. M., Beintema, D. A., Zijlstra, A. A., et al. 1998, A\&A, 331, 61

Wessson, R., \& Liu, X.-W. 2004, MNRAS, 351, 1026

Wisotzki, L., Köhler, T., Groote, D., et al. 1996, A\&AS, 115, 227

Wisotzki, L., Christlieb, N., Bade, N., et al. 2000, A\&A, 358, 77

Worseck, G., Fechner, C., Wisotzki, L., et al. 2007, A\&A, 473, 805

Yong, D., Lambert, D. L., \& Ivans, I. I. 2003, ApJ, 599, 1357

Yong, D., Aoki, W., \& Lambert, D. L. 2006, ApJ, 638, 1018

Zhang, Y., Liu, X.-W., Luo, S.-G., Péquignot, D., \& Barlow, M. J. 2005, A\&A, 442,249

Zoccali, M., Hill, V., Lecureur, A., et al. 2008, A\&A, 486, 177 